\title{
Myofilament Remodeling and Function Is More Impaired in Peripartum Cardiomyopathy Compared with Dilated Cardiomyopathy and Ischemic Heart Disease
}

Ilse A.E. Bollen, ${ }^{* \dagger}$ Elisabeth Ehler, ${ }^{\ddagger}$ Karin Fleischanderl,${ }^{\ddagger}$ Floor Bouwman, ${ }^{*}$ Lanette Kempers, ${ }^{*}$ Melanie Ricke-Hoch, Denise Hilfiker-Kleiner, ${ }^{\S}$ Cristobal G. dos Remedios, "Martina Krüger, " Aryan Vink, ${ }^{* *}$ Folkert W. Asselbergs, ${ }^{\dagger \dagger}+\ddagger \S \S$ Karin Y. van Spaendonck-Zwarts, ${ }^{\dagger \uparrow \uparrow}$ Yigal M. Pinto, ${ }^{\dagger \mid\|\|}$ Diederik W.D. Kuster, ${ }^{* \dagger}$ and Jolanda van der Velden ${ }^{* \dagger * * *}$

\begin{abstract}
From the Department of Physiology, ${ }^{*}$ VU University Medical Center, Amsterdam, the Netherlands; the Amsterdam Cardiovascular Sciences, ${ }^{\dagger}$ Amsterdam, the Netherlands; the Randall Division of Cell and Molecular Biophysics and Cardiovascular Division, ${ }^{\ddagger}$ British Heart Foundation Centre of Research Excellence, King's College London, London, United Kingdom; the Department of Cardiology and Angiology, ${ }^{\S}$ Hannover Medical School, Hannover, Germany; the Bosch Institute, "Discipline of Anatomy and Histology, University of Sydney, Sydney, New South Wales, Australia; the Institute of Cardiovascular Physiology, Heinrich-Heine University Düsseldorf, Düsseldorf, Germany; the Department of Pathology, ** University Medical Center Utrecht, Utrecht, the Netherlands; the Division Heart and Lungs, ${ }^{\dagger \dagger}$ Department of Cardiology, University of Utrecht, University Medical Center Utrecht, Utrecht, the Netherlands; the Durrer Center for Cardiogenetic Research, ${ }^{\ddagger \ddagger}$ Netherlands Heart Institute, Utrecht, the Netherlands; the Institute of Cardiovascular Science, ${ }^{\S \S}$ Faculty of Population Health Sciences, University College London, London, United Kingdom; the Department of Clinical Genetics ${ }^{\text {Tा }}$ and the Amsterdam Medical Center Heart Center, $\|\mid\|$ Department of Clinical and Experimental Cardiology, Academic Medical Center Amsterdam, University of Amsterdam, Amsterdam, the Netherlands; and the Netherlands Heart Institute, ${ }^{* * *}$ Utrecht, the Netherlands
\end{abstract}

\section{Accepted for publication} August 24, 2017.

Address correspondence to Ilse A.E. Bollen, M.Sc., Department of Physiology, VU University Medical Center, De Boelelaan 1117, Room 11W53, 1081 HV Amsterdam, the Netherlands. E-mail: a.bollen@vumc. nl.

\begin{abstract}
Peripartum cardiomyopathy (PPCM) and dilated cardiomyopathy (DCM) show similarities in clinical presentation. However, although DCM patients do not recover and slowly deteriorate further, PPCM patients show either a fast cardiac deterioration or complete recovery. The aim of this study was to assess if underlying cellular changes can explain the clinical similarities and differences in the two diseases. We, therefore, assessed sarcomeric protein expression, modification, titin isoform shift, and contractile behavior of cardiomyocytes in heart tissue of PPCM and DCM patients and compared these with nonfailing controls. Heart samples from ischemic heart disease (ISHD) patients served as heart failure control samples. Passive force was only increased in PPCM samples compared with controls, whereas PPCM, DCM, and ISHD samples all showed increased myofilament $\mathrm{Ca}^{2+}$ sensitivity. Lengthdependent activation was significantly impaired in PPCM compared with controls, no impairment was observed in ISHD samples, and DCM samples showed an intermediate response. Contractile impairments were caused by impaired protein kinase A (PKA)-mediated phosphorylation because exogenous PKA restored all parameters to control levels. Although DCM samples showed reexpression of EH-myomesin, an isoform usually only expressed in the heart before birth, PPCM and ISHD did not. The lack of EHmyomesin, combined with low PKA-mediated phosphorylation of myofilament proteins and increased compliant titin isoform, may explain the increase in passive force and blunted length-dependent activation of myofilaments in PPCM samples. (Am J Pathol 2017, 187: 2645-2658; https://doi.org/ 10.1016/j.ajpath.2017.08.022)
\end{abstract}

Supported by the Netherlands Cardiovascular Research Initiative, an initiative with support of the Dutch Heart Foundation, CVON2011-11 ARENA; the Rembrandt Institute for Cardiovascular Sciences 2013; British Heart Foundation; Dekker Scholarship-Junior Staff Member 2014T001 of the Dutch Heart Foundation; and UCL Hospitals NIHR Biomedical Research Centre.

Disclosures: None declared. 
Dilated cardiomyopathy (DCM) and peripartum cardiomyopathy (PPCM) are distinct forms of cardiac disease that share aspects of clinical presentation, such as dilated ventricle(s), reduced systolic function, and cardiac rhythm disorders. ${ }^{1,2}$ PPCM is a cardiac disease that presents itself during the last month of pregnancy, during delivery, or within the first 5 months postpartum. ${ }^{2}$ A genetic cause for both DCM and PPCM has been demonstrated before. $^{3-6}$ Evidence has accumulated showing that approximately $15 \%$ to $20 \%$ of PPCM patients carry cardiomyopathy-causing mutations, mainly in $T T N$, MYH7, and SCN5A, genes in which mutations are associated with DCM as well. ${ }^{3}$ PPCM patients are often young and show fast cardiac deterioration after disease onset, leading to the need for heart transplantation or death. ${ }^{7-10}$ In contrast, DCM patients develop cardiac disease at an older age, with a milder disease progression compared with PPCM patients. ${ }^{11}$ Although PPCM patients can show fast cardiac deterioration, they often can be stabilized or even show complete recovery on treatment. ${ }^{7-14}$ In a cohort of 182 PPCM patients in the United States, $27 \%$ showed full recovery, whereas $25 \%$ experienced at least one major adverse event (defined as death or complications that were life threatening or lead to long-term morbidity). ${ }^{15}$ Of the patients who survived a major adverse event, but did not receive a cardiac transplantation, 7 (32\%) experienced residual brain damage. Of the cohort, 13 patients died within 8 years, of whom 5 died suddenly, and 11 underwent cardiac transplantation within 2 years after diagnosis. Cardiopulmonary arrest occurred in six patients either during delivery or within the first 6 days after delivery. Severe pulmonary edema requiring intensive care occurred in 16 patients within 1 week after delivery. ${ }^{15}$ This cohort clearly illustrates the fast cardiac deterioration in PPCM patients. A German cohort of 115 PPCM patients reported an improvement rate of $85 \%$, a full recovery rate of $47 \%$, a failure to recover rate of $15 \%$, and a death rate of $2 \%,{ }^{13}$ demonstrating the high recovery rate from PPCM. ${ }^{13}$ DCM patients may be stabilized, but recovery after treatment is unlikely. ${ }^{6,14}$

Interestingly, DCM patients who become pregnant are more likely to experience adverse cardiac events, such as heart failure, sustained arrhythmia, or stroke, earlier in pregnancy ${ }^{16}$ than PPCM patients. This is likely caused by an increased hemodynamic load on an already troubled heart. In contrast, increased cardiac strain does not appear to be a disease trigger for PPCM because the highest incidence of disease onset is not at the time of the largest increase in hemodynamic load (approximately 24 weeks), but rather in the first month postpartum. ${ }^{10}$ In addition, it has been shown that the hormone prolactin plays a key role in PPCM pathogenesis. High levels of oxidative stress induce cleavage of the breastfeeding hormone prolactin into a $16-\mathrm{kDa}$ fragment that has detrimental effects on cardiac function. ${ }^{7,17,18}$ The peak of prolactin levels coincides with the peak incidence of disease onset in PPCM. Although prolactin levels are low in nonpregnant and nonnursing individuals, ${ }^{19}$ it is unlikely that prolactin plays a role in DCM. A recent study suggests detrimental effects of catecholamine treatment, specifically with the $\beta 1$-adrenergic receptor agonist dobutamine, in PPCM patients. ${ }^{13,20}$ In fact, experimental data showed that the $\beta 1$-receptor agonist isoproterenol impaired fatty acid and glucose uptake. ${ }^{20}$ The resulting energy depletion increased production of reactive oxygen species and led to cardiomyocyte death and progression of heart failure in STAT3 knockout mice, which could be attenuated with the $\beta$-blocker metoprolol. ${ }^{20}$ On the basis of differences in disease onset and progression, we hypothesize that different cellular remodeling underlies PPCM and DCM.

We studied myofilament functional and structural remodeling in PPCM and DCM compared with ischemic heart disease (ISHD) and nonfailing controls to define common and unique pathomechanisms for PPCM and DCM. We show that both PPCM and DCM patients experience increased myofilament $\mathrm{Ca}^{2+}$ sensitivity and reduced myofilament lengthdependent activation compared with controls. The changes in PPCM samples were more severe than observed in DCM samples, whereas ISHD samples only showed an increased $\mathrm{Ca}^{2+}$ sensitivity without an impairment in length-dependent activation. In addition, PPCM patients showed a significant increase in passive force $\left(\mathrm{F}_{\text {pass }}\right)$ development compared with controls, whereas DCM and ISHD samples showed similar $\mathrm{F}_{\text {pass }}$ as controls. Also, myofilament remodeling was different between DCM and PPCM patients. Although both patient groups showed a similar increase in compliant titin and fibrosis, only DCM patients expressed the EH-myomesin isoform, which could have contributed to the observed differences in contractile force of myofilaments. The lack of a stabilizing effect of EH-myomesin in PPCM may play a key role in the short-term fast disease progression observed in PPCM. On the other hand, limited remodeling might also explain total recovery in a large group of PPCM patients.

\section{Materials and Methods}

\section{Ethical Approval}

Left ventricular tissues from DCM, PPCM, and ISHD samples were acquired from the University of Sydney (Sydney, NSW, Australia), with the ethical approval of the Human Research Ethics Committee (number 2012/2814). The codes of used samples are as follows: DCM, 3.107, 4.036, 3.133, 4.125, 2.082, and 3.042; PPCM, 2.048, 3.058, 3.118, and 4.127; and ISHD, 4.111, 4.108, 4.070, and 4.091. Explanted left ventricular heart tissue of healthy donors were used as control samples; the donors died from a noncardiac cause, typically motor vehicle accidents. These healthy donor samples were also acquired from the University of Sydney, and samples included are as follows: 3.162, 6.042, 3.141, 3.164, 5.086, 6.034, 8.004, 7.040, 7.054, $6.008,5.128,7.044,4.104,6.020,3.160$, and 6.056. In addition, two DCM samples were acquired from the Biobank of the University Medical Center Utrecht (Utrecht, the Netherlands). This study was approved by the Biobank Research Ethics Committee, University Medical Center Utrecht (protocol number WARB 12/387). Written informed consent was obtained. Of the PPCM samples, two were acquired from the University of Hannover (Hannover, Germany). One sample was obtained during implantation of a left ventricular assist device during the 
acute phase of PPCM and the other after cardiac transplantation after chronic heart failure. PPCM tissue analyses were approved by the local ethics commission of the Hannover Medical School (Hannover, Germany); both patients provided written informed consent. All samples were stored in liquid nitrogen or at $-80^{\circ} \mathrm{C}$ until use.

\section{Fibrosis}

Cryosections ( $5 \mu \mathrm{m}$ thick) were stained with Picrosirius red. The amount of fibrosis was quantified using ImageJ version 1.49 (NIH, Bethesda, MD; http://imagej.nih.gov/ij). The fibrotic area was selected using the Huang thresholding method, threshold color red, color space red green blue, wide open blue and red filters, and adjusted green filter to distinguish stained from nonstained areas. The percentage of fibrosis was calculated as the percentage of red-stained tissue relative to the total area of the image analyzed. Of each sample, at least three images were taken, and data are shown as the average per sample.

\section{Cardiomyocyte Force Measurements}

Maximal force $\left(\mathrm{F}_{\max }\right)$ and $\mathrm{F}_{\text {pass }}$ of sarcomeres were measured at $\mathrm{pCa} 4.5$ and $\mathrm{pCa} 9.0$, respectively, in single membranepermeabilized cardiomyocytes mechanically isolated from heart tissue, as previously described. ${ }^{21,22}$ Briefly, a small piece of tissue (10 to $15 \mathrm{mg}$ ) was defrosted in $4^{\circ} \mathrm{C}$ isolation relax solution containing $1 \mathrm{mmol} / \mathrm{L}$ free $\mathrm{Mg}, 139.6 \mathrm{mmol} / \mathrm{L}$ $\mathrm{KCl}, 2 \mathrm{mmol} / \mathrm{L}$ EGTA, $5.95 \mathrm{mmol} / \mathrm{L}$ ATP, and $10 \mathrm{mmol} / \mathrm{L}$ imidazole, with the $\mathrm{pH}$ adjusted to 7.0 with $\mathrm{KOH}$. The tissue was cut in small pieces and mechanically disrupted with a Teflon piston for 5 to 10 seconds at $900 \times g$ to obtain a suspension of single cells, small clumps of cells, and cell fragments. To permeabilize the membranes, the cells were incubated with $0.5 \%$ Triton X-100 (Millipore, Burlington, MA) for 5 minutes at $4^{\circ} \mathrm{C}$. Cells were washed with isolation relax solution to remove Triton X-100 and kept at $4^{\circ} \mathrm{C}$ until measurement on the same day. Single cardiomyocytes were selected for measurement on the basis of size (100 to $150 \mu \mathrm{m}$ long and 10 to $35 \mu \mathrm{m}$ in diameter) and uniformity of striation pattern. Single cardiomyocytes were attached to stainless steel needles attached to a force transducer and a length motor with silicon-based glue (DB-025, Zwaluw; Den Braven, Oosterhout, the Netherlands) while being viewed with an inverted microscope at $\times 320$ magnification. Sarcomere length was determined by spatial Fourier transformation and set at the desired sarcomere length before force measurement, starting with the smallest sarcomere length. The relax $(\mathrm{pCa}$ 9.0) and activation ( $\mathrm{pCa} 4.5$ ) solutions contained 6.48 and $6.28 \mathrm{mmol} / \mathrm{L} \mathrm{MgCl}, 5.89$ and $5.97 \mathrm{mmol} / \mathrm{L} \mathrm{Na} 2 \mathrm{ATP}, 6.97$ and $0 \mathrm{mmol} / \mathrm{L}$ EGTA, and 0 and $7 \mathrm{mmol} / \mathrm{L} \mathrm{Ca}{ }^{2+}$-EGTA, respectively. Both relax and activation solution also contained $14.5 \mathrm{mmol} / \mathrm{L}$ creatine phosphate and $100 \mathrm{mmol} / \mathrm{L} \mathrm{N}, \mathrm{N}-$ bis[2-hydroxyethyl]-2-aminoethane-sulphonic acid, of which the $\mathrm{pH}$ was adjusted to 7.1 with $\mathrm{KOH}$. The ionic strength of the solutions was adjusted to $200 \mathrm{mmol} / \mathrm{L}$ with potassium propionic acid. The submaximal pCa solutions were generated by appropriate mixing of the relax and activation solutions. A single batch of $\mathrm{pCa}$ solutions was used for all active force measurements in this study to eliminate bias attributable to batch-to-batch variation. All passive and active force measurements were performed at $15^{\circ} \mathrm{C}$ to ensure stability of the cell during measurement, and temperature was controlled with a circulating water bath. For active force measurements, cells were transferred to the desired $\mathrm{pCa}\left(-\log \left[\mathrm{Ca}^{2+}\right]\right)$ and force development was recorded until steady state had been reached. Cells were then shortened with $30 \%$ of cell length to detach cross bridges and to determine total force development. Cells were then transferred back to a relax solution and shortened again with $30 \%$ of cell length to calculate passive force development. Active force development was calculated by subtraction of passive force from total force. Force was measured in activation solution ( $\mathrm{pCa} 4.5$ ) first to obtain $\mathrm{F}_{\max }$ development and thereafter at different $\mathrm{pCa}$ solutions in a random order. Finally, force was measured again in activation solution to confirm the cardiomyocyte did not have inappropriate reduction in $\mathrm{F}_{\max }$ during measurement protocol. Relative forces during the experiment were corrected for decrease in $\mathrm{F}_{\max }$ during the protocol, and measurements were excluded if the decrease in force was $>30 \%$. Passive force curves were measured by stretching the cell to various sarcomere lengths and measurement of passive force in relax solution. In a set of passive force measurements, 2,3-butanedione monoxime was used as an inhibitor for actinmyosin interaction at a concentration of $50 \mathrm{mmol} / \mathrm{L}$ in relax solution. All forces were normalized to cross-sectional area of the cardiomyocytes, calculated as (width $\times$ depth $\times \pi$ ) $/ 4$, at a sarcomere length of $2.3 \mu \mathrm{m}$. Protein kinase A (PKA) incubations were performed, as previously described. ${ }^{22}$ In short, membrane-permeabilized cardiomyocytes were incubated with $80 \mu \mathrm{L}$ of $1 \mathrm{U} / \mu \mathrm{L}$ PKA (P5511; Sigma, St. Louis, MO) with $0.006 \mathrm{mmol} / \mathrm{L}$ cAMP (Sigma). They were incubated in isolation relax solution at $20^{\circ} \mathrm{C}$ for 40 minutes before measurement of force at $15^{\circ} \mathrm{C}$, according to the same protocol described above, without prior PKA incubation. Relative force- $\left[\mathrm{Ca}^{2+}\right]$ curves were constructed. $\mathrm{Ca}^{2+}$ sensitivity was measured as the $\left[\mathrm{Ca}^{2+}\right]$ needed to achieve $50 \%$ of $\mathrm{F}_{\max }$ $\left(\mathrm{EC}_{50}\right)$, and length-dependent activation was measured as the shift in $\mathrm{EC}_{50}\left(\Delta \mathrm{EC}_{50}\right)$ at a sarcomere length of 1.9 and $2.3 \mu \mathrm{m}$. A representation of how $\mathrm{EC}_{50}$ and $\Delta \mathrm{EC}_{50}$ were calculated can be found in Supplemental Figure S1.

\section{Protein Expression and Modification}

Titin isoforms were separated on a $1 \%(\mathrm{w} / \mathrm{v})$ agarose gel and stained with SYPRO Ruby protein gel stain (Invitrogen, Carlsbad, CA), as described previously ${ }^{23}$; samples were measured in triplicate.

The size of N2BA titin was calculated, as described previously. ${ }^{24,25}$ In short, homogenates of the samples of interest (PPCM, DCM, and ISHD) were loaded adjacent to a nonfailing control heart and soleus muscle of an adult mouse on a $1 \%$ 
agarose gel. Proteins were separated in the same way as described above for titin isoform composition. A calibration curve was constructed on the basis of mobility of proteins of known molecular weight (N2A soleus, $3690 \mathrm{kDa}$; N2B nonfailing heart, $2970 \mathrm{kDa}$; nebulin soleus muscle, $748 \mathrm{kDa}$; and myosin heavy chain, $200 \mathrm{kDa}$ ) relative to myosin heavy chain. This calibration curve was used to calculate the molecular weight of the N2BA titin band of the sample of interest on the basis of relative mobility to myosin heavy chain. An example of a calibration curve and the associated calculation to determine titin N2BA size is depicted in Supplemental Figure S2, A and B. To avoid gel-to-gel variation or variation in mobility within a gel, only the adjacent lanes were used for the construction of the calibration curve. For all samples, the same reference control sample was used, and all samples were measured in duplicate.

To assess titin phosphorylation, homogenates were loaded on a $2.1 \%$ acrylamide, $0.5 \%$ agarose-strengthened gel and separated, as previously described. ${ }^{26}$ Proteins were transferred to a polyvinylidene difluoride membrane, blocked with $3 \%$ bovine serum albumin, and incubated with phosphorylation site-specific antibodies directed to Ser4010 (N2B unique sequence domain; PKA and extracellular signalregulated kinase 2 target) or Ser12022 (PEVK domain; protein kinase $\mathrm{C}$ and $\mathrm{Ca}^{2+} /$ calmodulin-dependent protein kinase II target), both from Eurogentec (Liège, Belgium). Incubation of primary antibodies was followed by incubation with secondary horseradish peroxidase-conjugated antibody. An enhanced chemiluminescence detection kit (Amersham; GE Healthcare Life Sciences, Chicago, IL) was used to detect phosphorylated proteins with the Fusion FX 7 (Vilber Lourmat, Collégien, France). Membranes were stripped to remove phosphorylation-specific antibody and blocked again with 3\% bovine serum albumin. Membranes were then incubated with an antibody directed to total titin (Eurogentec) and visualized with an enhanced chemiluminescence detection kit (Amersham).

Phosphorylations of cardiac troponin I (cTnI) and cardiac myosin binding protein $\mathrm{C}$ (cMyBP-C) were assessed, as previously described. ${ }^{27}$ Phosphorylation of $\mathrm{cTnI}$ was further studied by Phos-tag analysis, in which non-phosphorylated, monophosphorylated, and bis-phosphorylated cTnI samples (MA122700; Pierce, Appleton, WI) were separated by polyacrylamide-bound $\mathrm{Mn}^{2+}$-Phos-tag gel electrophoresis Western blotting, as previously described. ${ }^{28}$

Glutathionylation of cMyBP-C was assessed, as previously described, ${ }^{29}$ with minor deviations from protocol. In short: A nonreducing radioimmunoprecipitation assay buffer was used for protein isolation, to which $25 \mathrm{mmol} / \mathrm{L}$ N-ethylmaleimide (Sigma) was added before isolation. After protein isolation, the homogenate was diluted in a 1:1 ratio with $2 \times$ Laemmli sample buffer and loaded on Criterion TGX Precast gels, $8 \%$ to $16 \%$ (Bio-Rad Laboratories, Hercules, CA). Proteins were transferred to nitrocellulose membrane (Bio-Rad Laboratories) and blocked with 5\% blocking-grade buffer containing $2.5 \mathrm{mmol} / \mathrm{L}$ $\mathrm{N}$-ethylmaleimide. Membranes were cut and incubated with antibodies against glutathione (ab19534; Sigma) or glyceraldehyde-3-phosphate dehydrogenase (14C10; Cell Signaling, Danvers, MA). Membranes were stripped (Restore Western Blot Stripping Buffer; Thermo Fisher Scientific, Waltham, MA), blocked with 5\% blocking-grade buffer, and incubated with antibody against cMyBP-C (sc-67354; Santa Cruz Biotechnology, Dallas, TX), followed by incubation with secondary horseradish peroxidase-conjugated antibody. An enhanced chemiluminescence kit (Amersham) was used to detect proteins with the Amersham imager 600. As positive and negative controls for the glutathione Western blot, cardiomyocytes were isolated from a male Wistar rat (weight, 200 g). The animal experiments were performed in accordance with the guidelines from Directive 2010/63/EU of the European Parliament on the protection of animals used for scientific purposes. Liberase TM (Roche, Basel, Switzerland) ${ }^{30}$ was used for isolation of adult rat cardiomyocytes, and cells were suspended in plating medium containing medium 199 (Lonza, Basel, Switzerland), penicillin/streptomycin (1\%), and fetal bovine serum (5\%). Cardiomyocytes were carefully pipetted on laminin-coated glass coverslips. One hour after plating, cells that were not attached were removed by replacing the plating medium with culture medium that consisted of medium 199 , penicillin/streptomycin (1\%), and ITS supplement (insulin, 10 $\mathrm{mg} / \mathrm{L}$; transferrin, $5.5 \mathrm{mg} / \mathrm{L}$; and selenium, $5 \mu \mathrm{g} / \mathrm{L}$; Sigma Aldrich, St. Louis, MO). Positive controls were rat cardiomyocytes incubated for 1 hour with various concentrations of the membrane-permeable thiol-oxidizing agent diamide (Sigma). Negative controls were homogenates from rat cardiomyocytes treated with the reducing agents $100 \mathrm{mmol} / \mathrm{L}$ dithiothreitol and $1.4 \mathrm{~mol} / \mathrm{L} \beta$-mercaptoethanol before loading. The glutathione Western blot was confirmed to work because adding dithiothreitol and $\beta$-mercaptoethanol reduced the glutathione signal and adding diamide in nonreducing conditions increased the glutathione signal in a dose-dependent manner (Supplemental Figure S3).

\section{EH-Myomesin Expression Immunohistochemistry}

Human heart tissue pieces were cut on a Leica CM 1950 cryostat (Leica Biosystems, Wetzlar, Germany), and sections (12 $\mu \mathrm{m}$ thick) were retrieved on polylysine-coated slides and dried overnight. Sections were fixed for 5 minutes in acetone at $20^{\circ} \mathrm{C}$, followed by brief rehydration in phosphate-buffered saline, and 30 minutes blocking in 5\% preimmune goat serum (Sigma)/ $1 \%$ bovine serum albumin (Sigma) in gold buffer (20 $\mathrm{mmol} / \mathrm{L}$ Tris- $\mathrm{HCl}, \mathrm{pH} 7.5,155 \mathrm{mmol} / \mathrm{L} \mathrm{NaCl}, 2 \mathrm{mmol} / \mathrm{L}$ EGTA, and $2 \mathrm{mmol} / \mathrm{L} \mathrm{MgCl}_{2}$ ). Mouse monoclonal antimyomesin

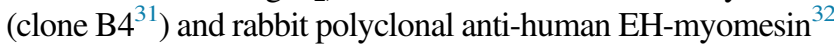
[generously donated by Dr. Irina Agarkova (Institute of Cell Biology, ETH Zurich, Switzerland)] were diluted in $1 \%$ bovine serum albumin/gold buffer and incubated overnight in a humid chamber. After $3 \times 5$ minutes wash in phosphate-buffered saline, secondary antibodies (Cy3-goat anti-mouse immunoglobulins and Cy2-goat anti-rabbit immunoglobulins; both multilabeling quality from Jackson Immunochemicals, West Grove, PA; via Stratech Scientific, Suffolk, UK) and DAPI were 
incubated for 30 minutes at room temperature in a humid chamber. After $3 \times 5$ minutes wash in phosphate-buffered saline, slides were mounted in Tris-buffered glycerol containing npropyl gallate ${ }^{32}$ with coverslips and were sealed with nail polish. Imaging was performed using a Leica SP5 confocal microscope (Leica Biosystems) equipped with a 405-nm blue diode and argon laser, a helium neon laser, and oil immersion lenses.

\section{EH-Myomesin Expression Western Blot}

After SDS-PAGE on a $4 \%$ to $8 \%$ TruPAGE Precast Gel (Sigma) at $120 \mathrm{~V}$, the human heart tissue samples were transferred overnight onto nitrocellulose (Amersham; GE Health Care) using a Bio-Rad Wet Blot system (Bio-Rad Laboratories) at 55 $\mathrm{mA}$. Transfer was visualized with Ponceau Red, followed by blocking in 5\% nonfat dry milk (Sainsbury's) in low-salt buffer for 1 hour. After incubation with primary antibody against human EH-myomesin ${ }^{32}$ and secondary antibody (horseradish peroxidase-conjugated goat anti-rabbit immunoglobulins; Calbiochem; Millipore), the chemiluminescence signal was visualized using Clarity Western ECL Substrate (Bio-Rad Laboratories) and detected with a Bio-Rad ChemiDoc Gel Imaging System (Bio-Rad Laboratories). For quantification, blots were reprobed with a polyclonal antibody against all actin isoforms (Sigma), and densitometry was performed using a Mathematica algorithm generously provided by Dr. Mark Holt (Randall Division, King's College London, London, UK).

\section{Statistical Analysis}

Graphpad Prism software version 7 (GraphPad Software Inc., San Diego, CA) was used for statistical analysis. $F_{\max }$ of patient cardiomyocytes was compared with control cardiomyocytes with one-way analysis of variance. $\mathrm{Ca}^{2+}$ sensitivity was calculated as $\mathrm{EC}_{50}$, and length-dependent activation was calculated as $\triangle \mathrm{EC}_{50}$. The amount of fibrosis, $\mathrm{EC}_{50}, \Delta \mathrm{EC}_{50}, \mathrm{~N} 2 \mathrm{BA} / \mathrm{N} 2 \mathrm{~B}$ ratio, phosphorylation of cTnI, and glutathionylation of cMyBP-C were compared with one-way analysis of variance. Phosphorylation of cMyBP-C was compared between groups with the nonparametric Kruskal-Wallis test. $\mathrm{F}_{\text {pass }}$ values in patient cardiomyocytes were compared with control cardiomyocytes by two-way analysis of variance. Correlations between N2BA/N2B and N2BA size, N2BA/N2B and fibrosis, and glutathionylation and phosphorylation of cMyBP-C were studied with linear regression. All values are shown as means \pm SEM. Statistical analysis was performed with Graphpad Prism version 7. $P<0.05$ was considered to represent a significant difference. $P$ values are indicated in figures versus controls.

\section{Results}

High $F_{\text {pass }}$ and Blunted Length-Dependent Activation of PPCM Myofilaments

The 16 control samples used for experiments were derived from 7 females and 9 males (age, $44.1 \pm 2.9$ years), the
PPCM samples were from 6 females (age, $35.7 \pm 3.2$ years), the DCM samples were from 3 females and 5 males (age, $50.0 \pm 2.9$ years), and the ISHD samples were from 4 males (age, $56.0 \pm 2.7$ years). To establish changes in functional remodeling of cardiomyocytes, myofilament force measurements were performed in membrane-permeabilized cardiomyocytes. $\mathrm{F}_{\max }$ was similar in all groups (Figure 1A). $\mathrm{F}_{\text {pass }}$ was significantly higher in PPCM samples compared with controls, and DCM and ISHD samples showed similar $\mathrm{F}_{\text {pass }}$ as controls (Figure 1B). This was not attributable to altered resting sarcomere length because this was similar in cardiomyocytes from all groups (Figure 1C). The same differences in $\mathrm{F}_{\text {pass }}$ between groups were obtained when measured in relax solution containing $50 \mathrm{mmol} / \mathrm{L}$ actin-myosin inhibitor 2,3-butanedione monoxime (Figure 1D), which implies the differences observed are caused by a passive myofilament component. $\mathrm{Ca}^{2+}$ sensitivity of myofilaments was significantly higher in all patient groups compared with controls, evident from the leftward shift of the force- $\mathrm{Ca}^{2+}$ relationship (Figure 1E). There was a significantly lower $\mathrm{EC}_{50}$ in PPCM, DCM, and ISHD samples compared with controls (Figure 1F). Length-dependent activation of myofilaments, indicated by the slope of the curve in Figure 1G, was lower in PPCM and DCM samples, but not in ISHD samples, compared with controls. The decrease in length-dependent activation, expressed as $\Delta \mathrm{EC}_{50}$, was largest in PPCM samples and significantly different from controls (Figure 1H).

\section{More Compliant Titin and Fibrosis in PPCM and DCM but Not in ISHD}

Titin is a giant sarcomeric protein that spans the entire sarcomere from the Z-disk to the M-band and is important in the regulation of $\mathrm{F}_{\text {pass }}$ through isoform switching and phosphorylation. ${ }^{33}$ In addition, it has been suggested that a switch to the more compliant titin isoform leads to reduced length-dependent activation. ${ }^{34,35}$ Titin can exist in the small and stiff $\mathrm{N} 2 \mathrm{~B}$ isoform and in longer and more compliant $\mathrm{N} 2 \mathrm{BA}$ isoforms. It has been shown that changes in titin isoform composition and phosphorylation contribute to DCM pathogenesis. ${ }^{36,37}$ However, it is unknown if titin isoform composition and phosphorylation is altered in PPCM patients. Titin isoforms were separated with gel electrophoresis (Figure 2A). Although PPCM samples showed a nonsignificant increase and DCM samples showed a significant increase in the N2BA/N2B ratio compared with controls, ISHD samples did not differ from controls (Figure 2B). The size of the N2BA titin was also increased in PPCM and DCM samples, whereas a smaller increase in N2BA size was observed in ISHD samples, compared with controls (Figure 2C). The size of the N2BA titin isoforms significantly correlated with the N2BA/N2B ratio in DCM and PPCM samples, but not in ISHD samples and controls (Figure 2D). This implies that there is more N2BA titin and also larger N2BA isoforms in PPCM and DCM samples. 
The increases in titin size and N2BA/N2B ratio imply that the heart induces a response to increase cellular compliance, possibly in response to stiffening of the heart by fibrosis. We, therefore, measured the amount of fibrosis by Picrosirius red staining in the same tissue samples in which $\mathrm{N} 2 \mathrm{BA} / \mathrm{N} 2 \mathrm{~B}$ ratio and titin size were measured. Figure $2 \mathrm{E}$ shows representative images of tissue samples in which fibrosis is stained in fierce red. A significant increase in fibrosis was found in PPCM and DCM samples compared with controls (Figure 2F), whereas the increase in fibrosis observed in ISHD samples was not significant compared with controls (Figure $2 \mathrm{~F}$ ). Figure $2 \mathrm{G}$ illustrates that a higher level of fibrosis coincides with higher N2BA/N2B ratios in PPCM and DCM samples.

\section{Reduced Phosphorylation and Preserved Glutathionylation of cMyBP-C in End-Stage Dilated Cardiomyopathies}

Oxidative stress has been shown to play an important role in PPCM pathogenesis and is also reported in other forms of heart failure ${ }^{7,38}$; it may underlie the changes in cellular function. Oxidative stress can cause glutathionylation of various proteins and thereby alter their function. ${ }^{39,40}$ Glutathionylation of cMyBP-C has been shown to impair phosphorylation of cMyBP-C and subsequently decelerate
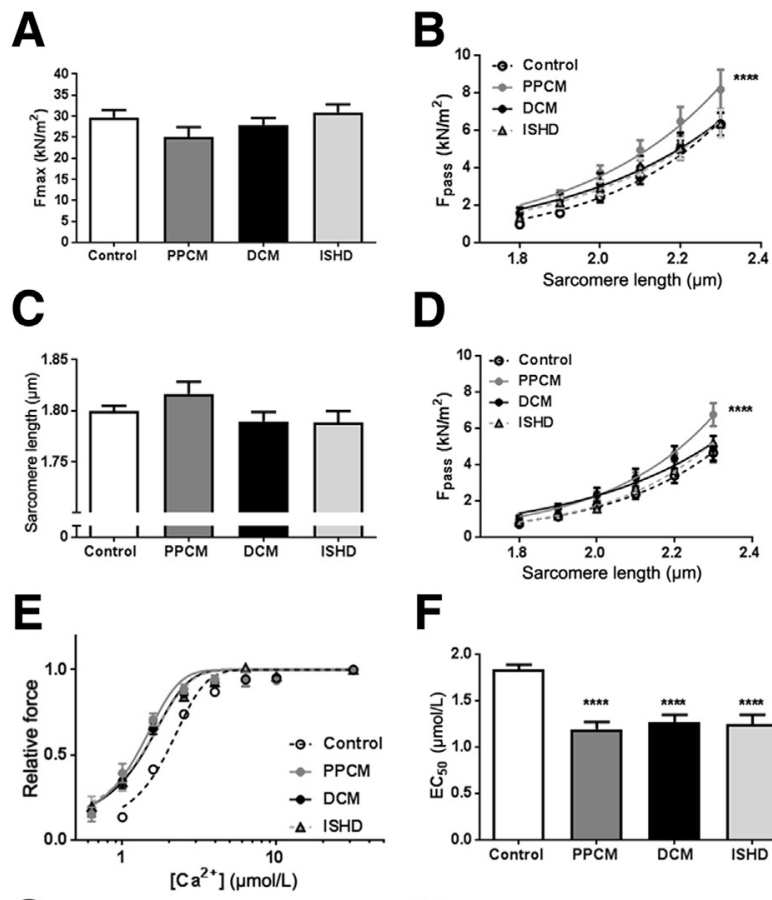

D

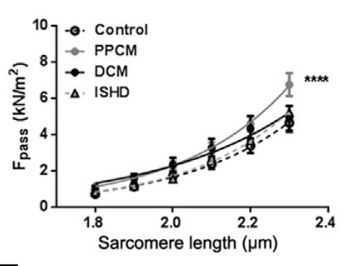

$\mathbf{F}$

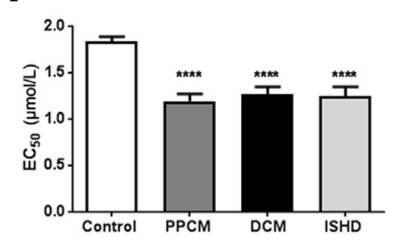

H

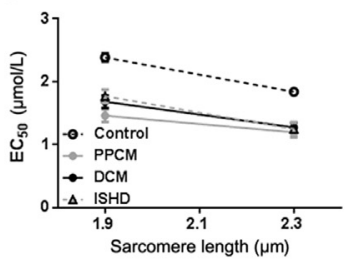

$\mathrm{Ca}^{2+}$-mediated force kinetics in DCM and ISHD. ${ }^{40}$ ProQstained (phosphorylation; Thermo Fisher Scientific) (Figure 3A) and SYPRO-stained (total protein) (Figure 3A) gel analysis showed decreased phosphorylation of cMyBP-C (Figure 3B) in PPCM, DCM, and ISHD samples compared with controls. Western blots for glutathionylated proteins (glutathionylated cMyBP-Cand total cMyBP-C) (Figure 3C) showed there was no significant increase in glutathionylated cMyBP-C (Figure 3D) in PPCM, DCM, and ISHD samples compared with controls. Also, no correlation was found between the amount of glutathionylated cMyBP-C and phosphorylation of cMyBP-C (Figure 3E). This implies that the decreased phosphorylation of cMyBP-C in these samples was not caused by glutathionylation of cMyBP-C. The decreased phosphorylation of cMyBP-C could have been caused by desensitization of the $\beta$-adrenergic receptors and subsequent impaired PKA-mediated phosphorylation, a mechanism confirmed in various forms of heart failure. ${ }^{41}$

\section{Restoration of cTnI and Titin Phosphorylation Normalized $\mathrm{F}_{\text {pass, }} \mathrm{Ca}^{2+}$ Sensitivity, and Length- Dependent Activation of Myofilaments}

Decreased PKA-mediated phosphorylation at Ser4010 of titin could underlie the higher $\mathrm{F}_{\text {pass }}$ in PPCM. ${ }^{42}$ Phosphorylation

\footnotetext{
Figure 1 Baseline characteristics. A: Maximal force $\left(F_{\max }\right)$ was not significantly different in PPCM $\left(25.1 \pm 2.3 \mathrm{kN} / \mathrm{m}^{2}\right), \mathrm{DCM}\left(27.9 \pm 1.7 \mathrm{kN} / \mathrm{m}^{2}\right)$, and ISHD $\left(30.9 \pm 1.9 \mathrm{kN} / \mathrm{m}^{2}\right)$, compared with controls $\left(29.7 \pm 1.8 \mathrm{kN} / \mathrm{m}^{2}\right)$. B: Passive force $\left(F_{\text {pass }}\right)$ was significantly increased in PPCM $(P<0.0001)$, compared with controls. $F_{\text {pass }}$ was not significantly altered in DCM and ISHD, compared with controls. C: Resting sarcomere length was not different between PPCM (1.82 \pm 0.012$),$ DCM (1.79 \pm 0.009$)$, ISHD (1.79 \pm 0.011$)$, and controls $(1.80 \pm 0.005) . \mathrm{D}: \mathrm{F}_{\text {pass }}$ measured in relax solution containing $50 \mathrm{mmol} / \mathrm{L} 2,3-$ butanedione monoxime showed significantly increased $F_{\text {pass }}$ in PPCM $(P=0.0001)$, compared with controls, whereas $\mathrm{F}_{\text {pass }}$ was not significantly altered in DCM and ISHD, compared with controls. E: The force-calcium curve was shifted to the left in PPCM, DCM, and ISHD samples, compared with controls. F: $\mathrm{Ca}^{2+}$ sensitivity, measured as $50 \%$ of $\mathrm{F}_{\max }\left(\mathrm{EC}_{50}\right)$, was significantly increased in PPCM $(1.19 \pm 0.08 \mu \mathrm{mol} / \mathrm{L}, P<0.0001), D C M(1.27 \pm 0.08 \mu \mathrm{mol} /$ $\mathrm{L}, P<0.0001)$, and ISHD $(1.25 \pm 0.10 \mu \mathrm{mol} / \mathrm{L}, P<0.0001)$, compared with controls $(1.84 \pm 0.05 \mu \mathrm{mol} / \mathrm{L})$. G: Length-dependent activation, indicated by the increase in $\mathrm{Ca}^{2+}$ sensitivity on stretch, was decreased in PPCM and DCM, but not in ISHD, compared with controls. $\mathbf{H}$ : The shift in $\mathrm{Ca}^{2+}$ sensitivity, indicated by $\triangle \mathrm{EC}_{50}$, was significantly reduced in PPCM $(0.26 \pm 0.05 \mu \mathrm{mol} / \mathrm{L}$, $P=0.0234)$, compared with controls $(0.54 \pm 0.08 \mu \mathrm{mol} / \mathrm{L})$, whereas it was nonsignificantly reduced in DCM $(0.41 \pm 0.03 \mu \mathrm{mol} / \mathrm{L})$ and similar to controls in $\operatorname{ISHD}(0.52 \pm 0.06 \mu \mathrm{mol} / \mathrm{L})$. Data are expressed as means \pm SEM. $n=4$ PPCM samples (A-C, E, F, and $\mathbf{H}) ; n=16$ PPCM cardiomyocytes $(\mathbf{A}) ; n=6 \mathrm{DCM}$ samples (A); $n=27$ DCM cardiomyocytes (A); $n=4$ ISHD samples (A-C, E, F, and $\mathbf{H}) ; n=15$ ISDH cardiomyocytes (A); $n=7$ control samples (A, B, E, F, and H); $n=30$ control cardiomyocytes (A); $n=14$ PPCM cardiomyocytes (B); $n=$ 6 DCM samples (B, C, E, F, and $\mathbf{H}) ; n=15$ DCM cardiomyocytes $(\mathbf{B}) ; n=9$ ISHD cardiomyocytes (B); $n=18$ control cardiomyocytes (B); $n=40$ PPCM cardiomyocytes (C); $n=36$ DCM cardiomyocytes (C); $n=22$ ISHD cardiomyocytes (C); $n=6$ control samples (C); $n=7$ control cardiomyocytes (C); $n=3$ PPCM samples (D); $n=13$ PPCM cardiomyocytes (D); $n=3$ DCM samples (D) $; n=8$ DCM cardiomyocytes (D); $n=3$ ISHD samples (D); $n=8$ ISHD cardiomyocytes (D); $n=4$ control samples (D); $n=11$ control cardiomyocytes (D); $n=8$ PPCM cardiomyocytes (E, F, and $\mathbf{H}) ; n=13$ DCM cardiomyocytes $(\mathbf{E}, \mathbf{F}$, and $\mathbf{H}) ; n=6$ ISHD cardiomyocytes $(\mathbf{E}, \mathbf{F}$, and $\mathbf{H}) ; n=17$ control cardiomyocytes $(\mathbf{E}, \mathbf{F}$, and $\mathbf{H})$. ${ }^{\star} P<0.05,{ }^{* \star * \star} P<0.0001$ versus controls.
} 

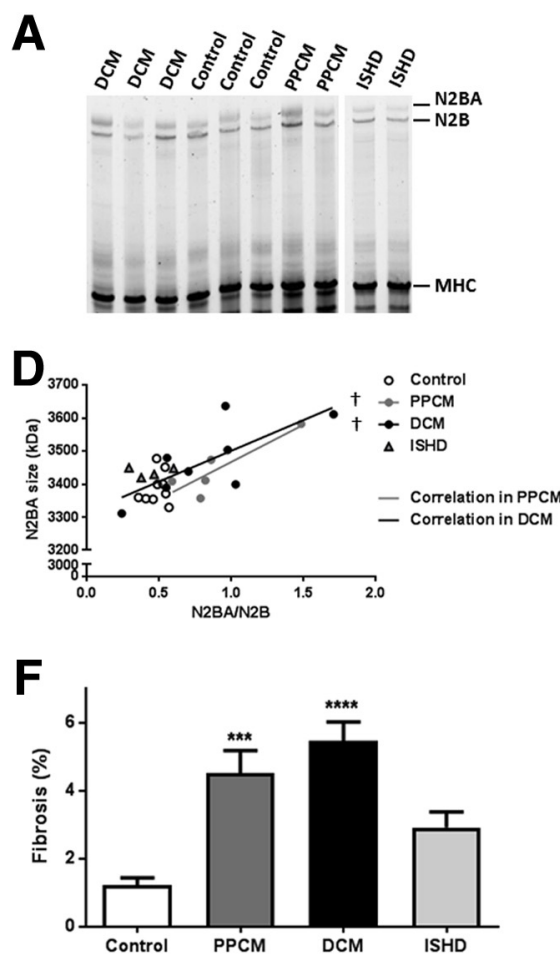
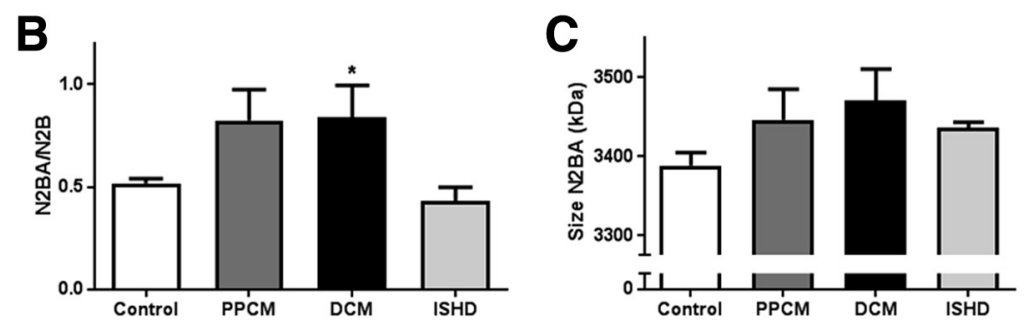

E
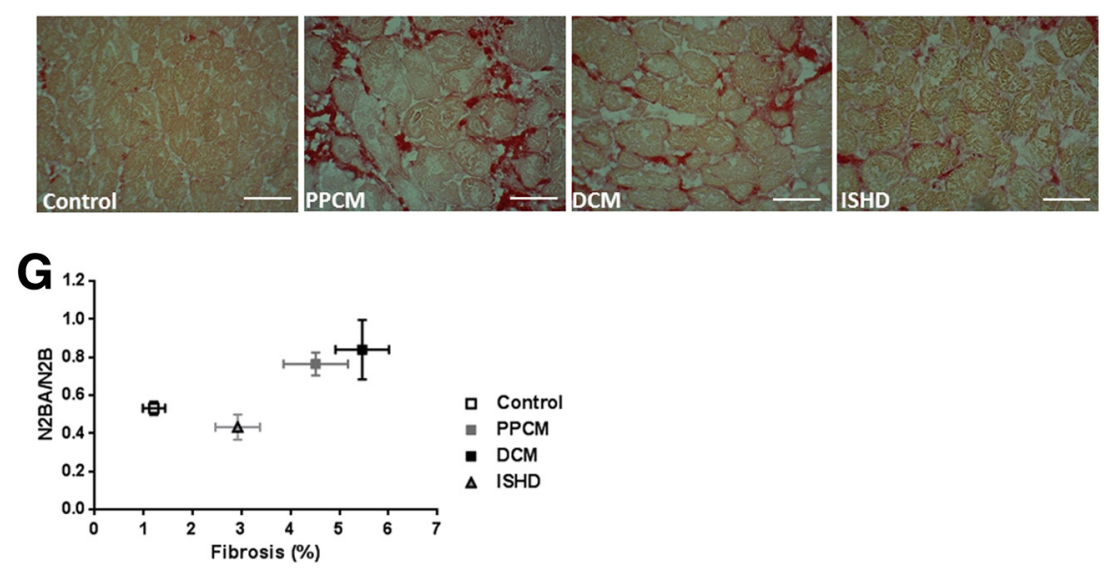

Figure 2 Titin isoform shift in DCM and PPCM and fibrosis in all dilated cardiomyopathies. A: Titin isoforms N2BA and N2B were separated with gel electrophoresis. B: N2BA/N2B was increased in DCM $(0.84 \pm 0.16, P=0.0327)$ and nonsignificantly in PPCM $(0.82 \pm 0.15)$, but not in ISHD (0.43 \pm 0.07$)$, compared with controls $(0.52 \pm 0.02)$. C: The size of N2BA titin was nonsignificantly increased in DCM (3471 $\pm 39 \mathrm{kDa})$ and PPCM (3446 $\pm 39 \mathrm{kDa})$, compared with controls (3388 $\pm 16 \mathrm{kDa}$ ), whereas a smaller increase in N2BA size was observed in ISHD (3436 $\pm 7 \mathrm{kDa})$. D: The size of N2BA was significantly correlated to the N2BA/N2B ratio in PPCM $\left(P=0.0479, R^{2}=0.7775\right)$ and DCM $\left(P=0.0365, R^{2}=0.5450\right)$, but not in ISHD, controls, or all groups combined. E: Representative images of cryosections stained with Picrosirius red of a control, PPCM, DCM, and ISHD sample. F: Fibrosis was significantly increased in PPCM $(4.51 \% \pm 0.66 \%, P=0.0003)$ and DCM $(5.46 \% \pm 0.55 \%, P<0.0001)$, compared with controls $(1.21 \% \pm 0.23 \%)$, whereas fibrosis was only nonsignificantly increased in ISHD $(2.92 \% \pm 0.46 \%)$. G: No significant correlation was found between the amount of fibrosis and N2BA/N2B. Data are expressed as means \pm SEM. $n=6$ PPCM samples (B); $n=8$ DCM samples (B, C, and F); $n=4$ ISHD samples (B, C, and F); $n=15$ control samples (B); $n=5$ PPCM samples (C); $n=9$ control samples $(\mathbf{C}$ and $\mathbf{F}) ; n=4$ PPCM samples $(\mathbf{F}) .{ }^{*} P<0.05,{ }^{* * *} P<0.001$, and ${ }^{* * * *} P<0.0001$ versus controls; ${ }^{\dagger} P<0.05$ N2BA/N2B ratio versus $\mathrm{N} 2 \mathrm{BA}$ size (slope of linear regression). Scale bars $=50 \mu \mathrm{m}(\mathbf{E})$.

of Ser4010 was decreased in PPCM samples compared with controls (Figure 4, A and C). However, Ser4010 phosphorylation was also decreased in DCM samples (Figure 4, A and C), whereas $F_{\text {pass }}$ was not altered in DCM samples. An alternative hypothesis for the discrepant change in $\mathrm{F}_{\text {pass }}$ in PPCM and DCM would be that protein kinase $\mathrm{C}-$-mediated phosphorylation of titin is increased in PPCM, leading to an increased $\mathrm{F}_{\text {pass. }}{ }^{42}$ However, protein kinase $\mathrm{C}$-mediated phosphorylation at Ser12022 was decreased, rather than increased, in PPCM, and unaltered in DCM and ISHD compared with controls (Figure 4, B and D). To determine whether the decrease in Ser4010 phosphorylation was causal to the increase in $\mathrm{F}_{\mathrm{pass}}$ in PPCM samples, the experiments were repeated after incubation with exogenous PKA. Indeed, PKA normalized $F_{\text {pass }}$ in PPCM samples (Figure 4E). Impaired PKA-mediated phosphorylation could also cause hypophosphorylation of cTnI, which is an important regulator of myofilament $\mathrm{Ca}^{2+}$ sensitivity and length-dependent activation. ${ }^{43,44}$ Indeed, cTnI phosphorylation was also decreased in PPCM, DCM, and ISHD samples compared with controls (Figures 3A and 4F). A Phos-tag analysis of
cTnI showed prominent monophosphorylation and bisphosphorylation of cTnI in controls, whereas PPCM and DCM samples showed prominent non-phosphorylation and monophosphorylation of cTnI. The ISHD samples showed predominantly monophosphorylated cTnI (Figure 4, G and $\mathrm{H})$. Exogenous PKA normalized myofilament $\mathrm{Ca}^{2+}$ sensitivity in PPCM, DCM, and ISHD samples to control levels (Figure 4, I and J). In addition, exogenous PKA restored $\triangle \mathrm{EC}_{50}$ in PPCM and DCM to control levels (Figure $4 \mathrm{~K}$ ).

\section{Expression of EH-Myomesin in DCM, but Not in PPCM and ISHD, Samples}

Although $\mathrm{F}_{\text {pass }}$ normalized to control levels after PKA in the PPCM samples, we set out to find an explanation for the discrepant finding of $\mathrm{F}_{\text {pass }}$ and titin phosphorylation in PPCM, DCM, and ISHD samples. Myomesin is a component of the Mband and is important for sarcomere stability. The fetal form of myomesin, EH-myomesin, has been shown to be a hallmark of DCM and is virtually absent in adult healthy heart tissue. ${ }^{32}$ It is believed that $\mathrm{EH}$-myomesin is reexpressed to provide stability in 

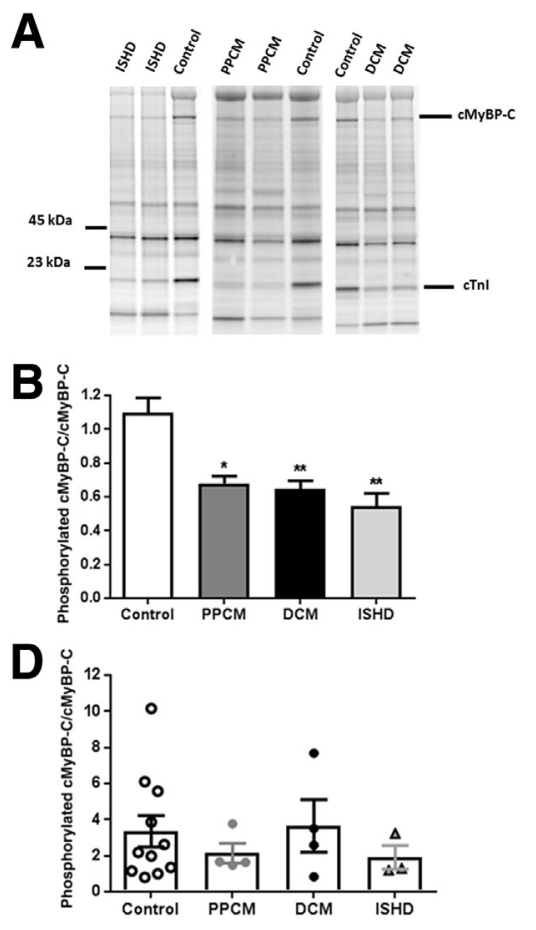
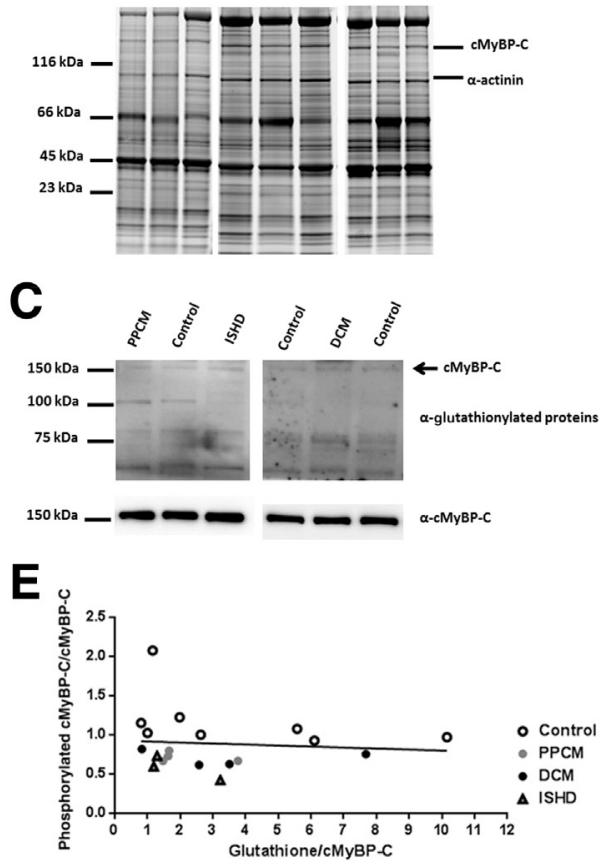

Figure 3 Glutathionylation and phosphorylation of cardiac myosin binding protein C (CMyBPC). A: Left panel: Gel stained with ProQ diamond. Right panel: Same gel stained with SYPRO Ruby. B: Phosphorylation of CMyBP-C was significantly decreased in PPCM $(P=0.0125)$, DCM $(P=0.0027)$, and ISHD $(P=0.0011)$, compared with controls. C: Representative blots stained with $\alpha$-glutathione (top panel) and CMyBP-C (bottom panel). D: Glutathionylation of CMyBP-C was not different in PPCM, DCM, and ISHD, compared with controls. E: No correlation was found between gluthationylation and phosphorylation of CMyBPC. Data are expressed as means \pm SEM. $n=5$ PPCM samples (B); $n=6$ DCM samples (B); $n=4$ ISHD samples (B); $n=13$ control samples (B); $n=4$ PPCM samples (D); $n=4$ DCM samples (D); $n=3$ ISHD samples (D); $n=3$ ISHD samples (D); $n=11$ control samples (D). ${ }^{*} P<0.05,{ }^{*}{ }^{*} P<0.01$ versus controls. cTnI, cardiac troponin I. overstretched conditions in DCM. Our study confirmed EHmyomesin was reexpressed in DCM samples (Figure 5, C and G). Expression of EH-myomesin was much lower in ISHD samples (Figure 5, D and $\mathrm{H}$ ) and almost absent in PPCM samples (Figure 5, B and F). Our control patients did not show expression of EH-myomesin or only showed expression at a low level (Figure 5, A and E). Western blot analysis confirmed high EH-myomesin expression in DCM, but not in PPCM and controls (Figure 5, I and J). Thus, the increase in titin N2BA/N2B ratio is accompanied by an increase in EH-myomesin in DCM, but not in PPCM. The absence of the stabilizing effect of EHmyomesin expression in PPCM may provide an explanation about why PPCM showed more impairment of myofilament function compared with DCM. Titin isoform expression and PKA-mediated myofilament phosphorylation were similar in the two groups.

\section{Discussion}

The clinical similarities and differences in disease onset, progression, and outcome between PPCM and DCM patients suggest that the cellular pathomechanisms overlap, but may also show distinct changes. Herein, we show that myofilament function is more impaired in PPCM compared with DCM with respect to length-dependent activation and $\mathrm{F}_{\text {pass. }}$ In addition, myofilament remodeling was different, because DCM samples expressed EH-myomesin, whereas PPCM samples did not. On the other hand, PPCM and DCM samples showed similar changes in myofilament $\mathrm{Ca}^{2+}$ sensitivity and sarcomeric protein phosphorylation.
Fibrosis Content Is Higher in PPCM and DCM Compared with ISHD and Controls

Although fibrosis is consistently reported in $\mathrm{DCM},{ }^{45,46}$ reports on the occurrence of fibrosis in PPCM patients are conflicting $^{47-50}$; animal models of PPCM show fibrosis. ${ }^{51}$ Magnetic resonance imaging data at diagnosis of PPCM in patients frequently display no hint for fibrosis, a feature that would be consistent with the ability of many patients to fully recover from the disease. ${ }^{17,52}$ In the present study, we found fibrosis in both DCM and PPCM samples. However, the assessment of fibrosis was performed in small tissue areas of samples obtained during heart transplantation. It cannot be excluded that the tissue samples were selectively taken from more fibrotic regions and may, thus, not be representative of whole heart fibrosis content. Techniques, such as magnetic resonance imaging, might give a better impression of whole heart fibrosis content. No late gadolinium enhancement by magnetic resonance imaging was shown in PPCM (two acute phase patients and four patients at a later stage) previously. ${ }^{49}$ In addition, our fibrosis analysis was only performed in end-stage explanted heart tissue of PPCM patients and might, therefore, have been induced over time and may not reflect the acute or short-term PPCM situation. The ISHD samples were taken from the remote area and not within the infarct zone. Although PPCM and DCM are more likely to show a diffuse fibrosis pattern and, therefore, any part of the heart taken might show fibrosis, the fibrosis in ISHD is more likely to be localized in or close to the infarct area. This could explain why we found little fibrosis in the ISHD samples. 
A

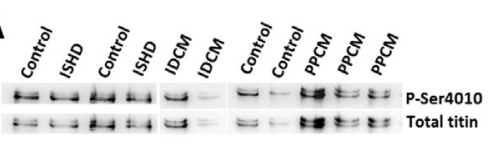

C
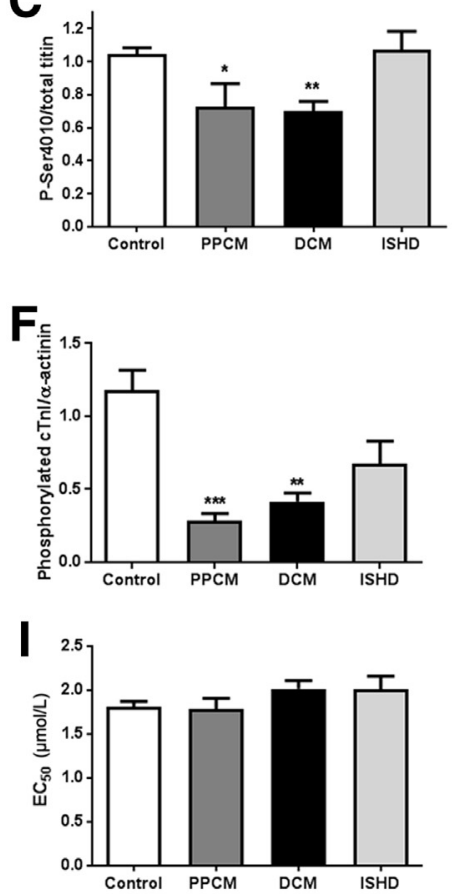

$B_{8}$

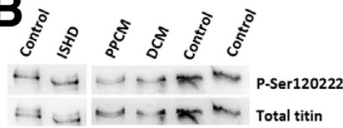

D

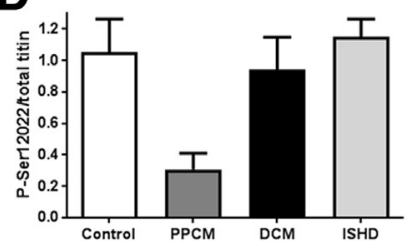

G
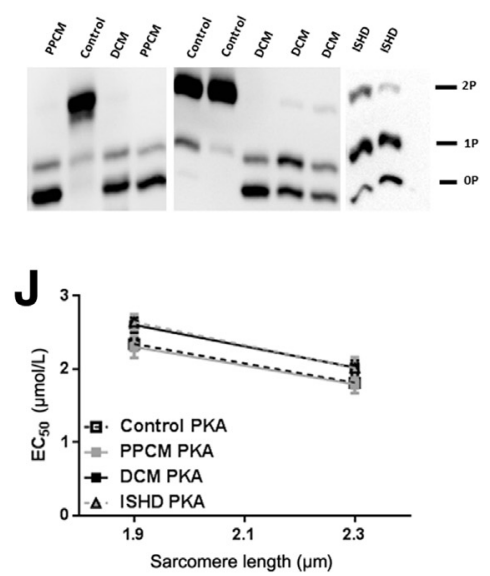

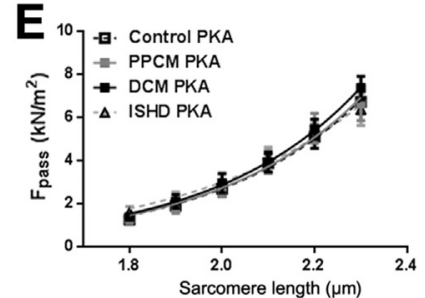

H
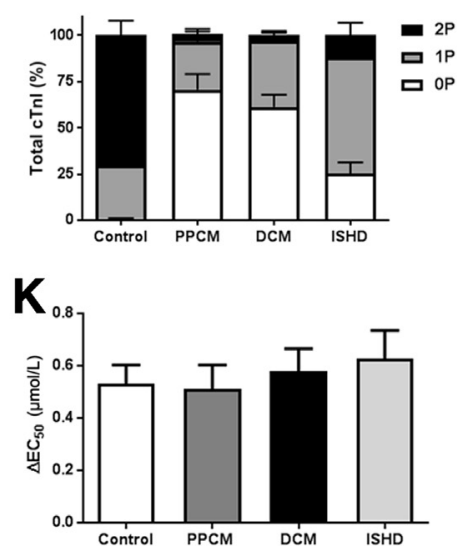

Figure 4 rotein kinase A (PKA)-mediated hypophosphorylation in PPCM, DCM, and ISHD. A: Representative images of phosphorylated titin at Ser4010 (top row) and total titin (bottom row). B: Representative images of phosphorylated titin at Ser12022 (top row) and total titin (bottom row). C: PKAmediated phosphorylation of titin at Ser4010 was significantly reduced in PPCM $(P=0.0140)$ and DCM $(P=0.0051)$ samples, compared with controls, whereas it was not altered in ISHD samples. D: Protein kinase C-mediated phosphorylation of titin at Ser12022 was nonsignificantly decreased in PPCM but normal in DCM and ISHD samples, compared with controls. E: Passive force $\left(F_{\text {pass }}\right)$ normalized to controls after incubation with exogenous PKA in PPCM samples, whereas PKA did not alter $\mathrm{F}_{\text {pass }}$ in DCM or ISHD samples. F: Phosphorylation of cardiac troponin I (cTnI) was significantly reduced in PPCM $(P=0.0004)$ and $\operatorname{DCM}(P=0.001)$, and nonsignificantly reduced in ISHD $(P=0.0681)$, samples, compared with controls. G: Phos-tag analysis showed separation of non-phosphorylation (OP), monophosphorylation (1P), and bis-phosphorylation (2P) of cTnI. H: Phos-tag analysis showed predominantly monophosphorylation and bis-phosphorylation of CTnI in controls, whereas non-phosphorylation and monophosphorylation were more prevalent in PPCM and DCM. ISHD samples showed mostly monophosphorylation of cTnI. I: Incubation with exogenous PKA normalized $\mathrm{Ca}^{2+}$ sensitivity in PPCM (1.79 \pm 0.12$)$, DCM $(2.02 \pm 0.09)$, and ISHD $(2.01 \pm 0.15)$ samples, compared with controls $(1.81 \pm 0.06)$. J: Length-dependent activation, indicated by the increase in Ca ${ }^{2+}$ sensitivity on stretch, was normalized after incubation with exogenous PKA to controls in PPCM, DCM, and ISHD samples, compared with controls. K: The shift in $\mathrm{Ca}^{2+}$ sensitivity on stretch, indicated by $\triangle \mathrm{EC}_{50}$, was normalized to controls after incubation with exogenous PKA in PPCM (0.51 \pm 0.09$), \mathrm{DCM}(0.58 \pm 0.08)$, and ISHD $(0.63 \pm 0.11)$ samples, compared with controls $(0.53 \pm 0.07)$. Data are expressed as means \pm SEM. $n=5$ PPCM samples $(\mathbf{C}, \mathbf{F}$, and $\mathbf{H}-\mathbf{K}) ; n=6$ DCM samples (C, D, F, and $\mathbf{H}) ; n=4$ ISHD samples (C, E, F, and $\mathbf{I}-\mathbf{K}) ; n=12$ control samples (C); $n=4$ PPCM samples (D and E); $n=3$ ISHD samples (D and $\mathbf{H}) ; n=11$ control samples (D); $n=11$ PPCM cardiomyocytes (E); $n=6$ DCM samples (E and $\mathbf{I}-\mathbf{K}) ; n=11$ DCM cardiomyocytes (E); $n=10$ ISHD cardiomyocytes $(\mathbf{E}) ; n=7$ control samples $(\mathbf{E}) ; n=14$ control cardiomyocytes $(\mathbf{E}) ; n=13$ control samples $(\mathbf{F}) ; n=8$ control samples $(\mathbf{H}) ; n=8$ PPCM cardiomyocytes (I-K); $n=8$ DCM cardiomyocytes (I-K); $n=6$ ISHD cardiomyocytes (I-K); $n=6$ control samples (I-K); $n=17$ control cardiomyocytes (I-K). ${ }^{*} P<0.05,{ }^{*} P<0.01$, and ${ }^{* * *} P<0.001$ versus controls.

\section{PKA-Mediated Hypophosphorylation in All Dilated Cardiomyopathies}

Independent of the initial cause, all forms of dilated cardiomyopathy (PPCM, DCM, and ISHD) exhibited higher myofilament $\mathrm{Ca}^{2+}$ sensitivity compared with controls, whereas only PPCM samples had an increased $F_{\text {pass. }}$. The increase in myofilament $\mathrm{Ca}^{2+}$ sensitivity was attributed to decreased PKA-mediated phosphorylation of cTnI because incubation with exogenous PKA restored $\mathrm{Ca}^{2+}$ sensitivity to controls in all groups. The increased $\mathrm{F}_{\text {pass }}$ in PPCM samples was also restored with exogenous PKA, and a decreased PKA-mediated phosphorylation of titin was confirmed at Ser4010. In addition, we also found a decreased phosphorylation of cMyBP-C, which was not related to glutathionylation of cMyBP-C. These combined results strengthen the suggestion that PKA-mediated phosphorylation was impaired in all heart failure samples. The $\beta$ adrenergic receptors were shown to be desensitized, leading to impaired PKA-mediated phosphorylation of sarcomeric proteins in various forms of heart failure. ${ }^{41} \mathrm{We}$ have now confirmed PKA-mediated phosphorylation is impaired in 


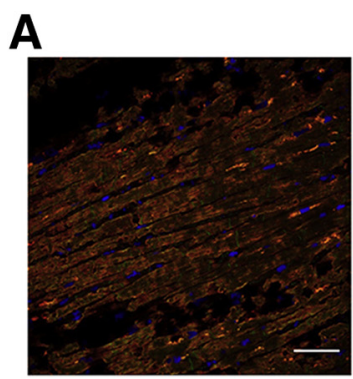

Controls

E

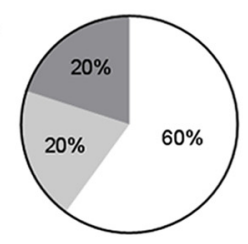

B

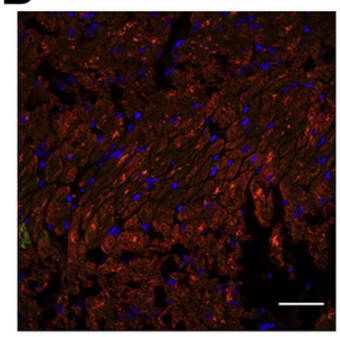

PPCM

F

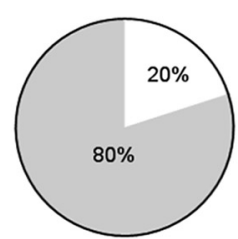

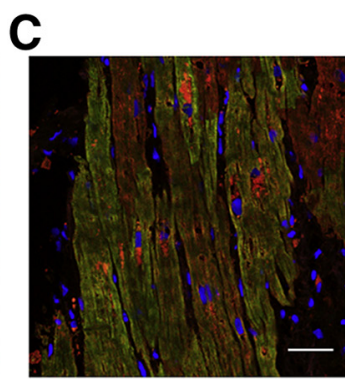

DCM

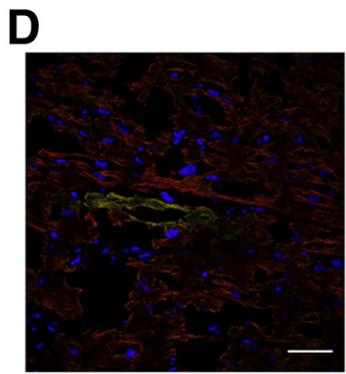

ISHD
G

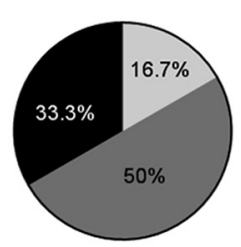

H

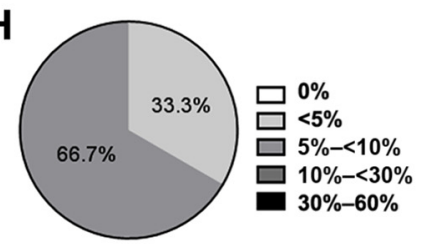

I

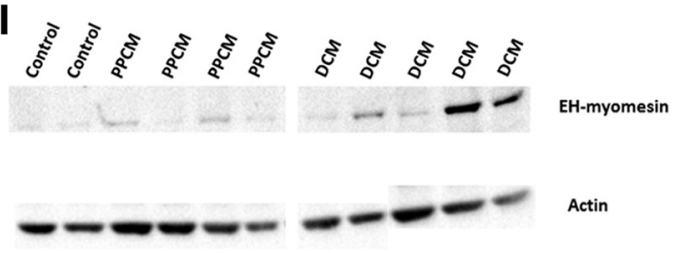

J

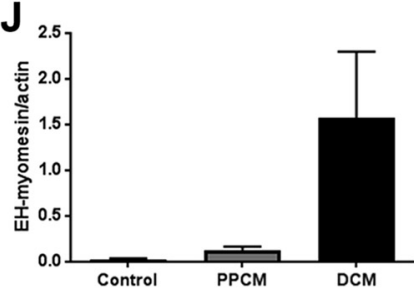

Figure 5 EH-myomesin expression in DCM, but only limited in PPCM and ISHD, compared with controls. A-D: Representative immunohistochemical images of controls (A), PPCM (B), DCM (C), and ISHD (D), in which EH-myomesin is stained in green, all myomesin isoforms in red, and nuclei in blue. E-H: The percentage of patients in control (E), PPCM (F), DCM (G), and ISHD (H) groups that fall in various categories of degree of EH-myomesin expression is indicated in pie charts. I and $\mathbf{J}$ : Western blot showed EH-myomesin expression (top panel) relative to actin (bottom panel; I) is nonsignificantly increased in DCM, compared with PPCM and controls (J). Data are expressed as means \pm SEM. $n=4$ PPCM and DCM samples (I); $n=2$ control samples (I). Scale bars $=50 \mu \mathrm{m}$.

end-stage PPCM as well. Despite a similar down-regulation of cTnI phosphorylation, the $\Delta \mathrm{EC}_{50}$ was significantly decreased only in PPCM samples compared with controls. Although DCM samples showed a nonsignificant impairment of length-dependent activation, ISHD samples had normal length-dependent activation values compared with controls, despite their increased $\mathrm{Ca}^{2+}$ sensitivity. Decreased length-dependent activation has been reported for samples with compliant titin, ${ }^{35,53}$ and an increase in compliant titin has been reported in DCM. ${ }^{36,37}$ We observed an increase in the N2BA/N2B ratio in PPCM and DCM samples compared with controls. In addition, an increase in size of the compliant titin isoform was found, corresponding to $58 \mathrm{kDa}$ in PPCM, $93 \mathrm{kDa}$ in DCM, and $48 \mathrm{kDa}$ in ISHD samples, compared with controls. Although this increase was not significant, it is of a magnitude that should not be ignored because such an increase in molecular weight might alter protein function. The fact that titin N2BA size and the N2BA/N2B ratio were significantly correlated in DCM and PPCM samples indicates that the same regulatory mechanism is responsible for both the shift in isoform and the inclusion of additional exons to make N2BA bigger. This observation is in line with the increase in titin N2BA size and $\mathrm{N} 2 \mathrm{BA} / \mathrm{N} 2 \mathrm{~B}$ ratio found in a patient with a mutation in
RBM20. ${ }^{24}$ The observation that in ISHD, N2BA size was increased, whereas N2BA/N2B ratio was unaltered, implies the isoform shift is preceded by inclusion of additional exons in N2BA, after which both titin size and N2BA expression increase further. Both $\mathrm{cTnI}$ phosphorylation and titin isoform composition are regulators of myofilament length-dependent activation. ${ }^{34}$ The decreased cTnI phosphorylation and increased N2BA/N2B and N2BA size explain the blunted myofilament length- dependent activation in PPCM and DCM samples. The preserved myofilament length-dependent activation in ISHD samples, which showed decreased cTnI phosphorylation and unaltered N2BA/N2B ratio, indicates a synergistic role of cTnI phosphorylation and titin in regulating myofilament lengthdependent activation.

Different Myofilament Remodeling of the M-Band in PPCM and DCM

PPCM and DCM samples both had decreased phosphorylation at Ser4010 of titin and a similar increase in N2BA/N2B ratio, whereas $F_{\text {pass }}$ was higher compared with controls only in PPCM. The expression of myomesin isoforms follows the expression of titin isoforms in various muscle types. The 


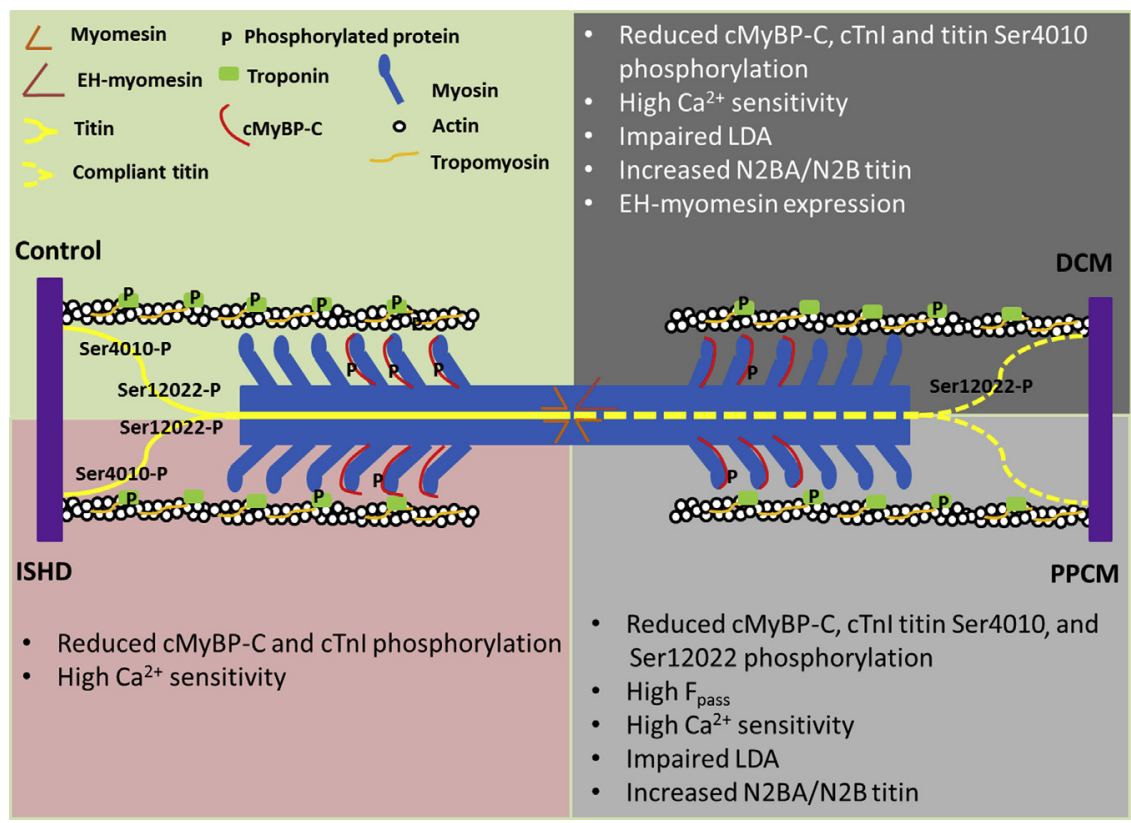

Figure 6 Overview of pathological changes in PPCM, DCM, and ISHD. All dilated cardiomyopathies showed a decreased cardiac myosin binding protein $\mathrm{C}(\mathrm{CMyBP}-\mathrm{C})$ phosphorylation and an increased $\mathrm{Ca}^{2+}$ sensitivity induced by decreased phosphorylation of cardiac troponin I (cTnI). However, PPCM and DCM share additional pathomechanisms, such as increased compliant titin, reduced phosphorylation of titin at Ser4010, and impaired length-dependent activation. Phosphorylation of titin Ser12022 was only decreased in PPCM. Although DCM samples showed reexpression of EH-myomesin, PPCM and ISHD samples did not. The lack of EH-myomesin reexpression in PPCM in combination with low cTnI phosphorylation and enhanced compliant titin isoform expression may explain high passive force $\left(F_{\text {pass }}\right)$ and the more pronounced myofilament length-dependent activation impairment in PPCM. LDA, length-dependent activation. longer compliant isoforms of titin and EH-myomesin are predominantly expressed in the fetal heart and are replaced by the shorter and stiffer myomesin during the progression to adulthood. ${ }^{54,55}$ This was also reported for soleus, where the larger titin $\mathrm{N} 2 \mathrm{~A}$ isoform $^{56}$ is expressed, and the $\mathrm{EH}-$ myomesin dominates. ${ }^{57}$ The reexpression of EH-myomesin in DCM patients could be a result of the induction of the fetal gene program, which has been shown in various forms of heart failure and is believed to be activated to cope with the changes in cardiac demand. ${ }^{58}$ Whether the expression of these fetal genes is beneficial is still under debate. EHmyomesin acts as a minispring and has an additional domain of approximately 200 amino acids, similar to the PEVK domain of titin. ${ }^{59}$ It is believed that EH-myomesin provides stability to the sarcomere in overstretched conditions. ${ }^{59}$ Herein, we presented evidence that expression of $\mathrm{EH}-$ myomesin might prevent an increase in $\mathrm{F}_{\text {pass }}$ and a decrease of length-dependent activation in DCM patients, but not in PPCM patients, who do not express EH-myomesin. Exogenous PKA restored $\mathrm{F}_{\text {pass }}$ and length-dependent activation in PPCM samples, indicating that the lack of EH-myomesin is overcome by PKA-mediated protein phosphorylation. An in vitro study showed phosphorylation of myomesin at Ser482 by PKA and subsequent disturbance of the interaction between titin (through the M4 domain) and myomesin (the My4 to My6 domains). ${ }^{60}$ However, Fukuzawa et al ${ }^{61}$ could not confirm a titin (M4)-myomesin (My4 to My6) interaction in a pulldown assay or a forced Yeast Two-Hybrid assay. In addition, phosphorylation of myomesin did not affect the myomesin-obscurin interaction. Therefore, the effect of myomesin phosphorylation in vivo warrants further research. Nonetheless, in heart failure, where the $\beta$-adrenergic receptors are desensitized and PKA-mediated phosphorylation of sarcomeric proteins, such as cTnI and titin, is decreased, the expression of EH-myomesin may play an important role in stabilization of the sarcomeres to maintain or limit the increase in $\mathrm{F}_{\text {pass }}$ and impairment of length-dependent activation.

\section{Clinical Perspective}

Although PPCM and DCM have similar clinical phenotypes, they differ in their clinical progression and, therefore, have a different prognosis. We show herein that myofilament remodeling is different in PPCM and DCM. We also show that PPCM samples have impaired PKA-mediated phosphorylation, similar to what has been observed in DCM samples, but that the effect on myofilament function is larger in PPCM. These observations could provide a possible explanation about why PPCM patients have such a strong response to stimulation of their $\beta$-adrenergic system. ${ }^{20}$ The notion that we could restore all parameters with exogenous PKA indicates that both PPCM and DCM patients might benefit from an increase in $\beta$-adrenergic stimulation to normalize myofilament function. However, we have discussed before that dobutamine, a $\beta$-adrenergic receptor agonist, worsens heart failure in PPCM patients and STAT3 knockout mice, and is associated with adverse outcome independent of treatment with bromocriptine. ${ }^{13,20}$ These results question whether the $\beta$-adrenergic system is, therefore, a realistic clinical target in PPCM treatment. However, increasing $\mathrm{Ca}^{2+}$ sensitivity has been a target for the treatment of systolic dysfunction because a greater force is generated at lower calcium concentrations to achieve sufficient contractile power in systolic dysfunction. We show that the myofilaments are already very $\mathrm{Ca}^{2+}$ sensitive and this should be taken into consideration during treatment. In addition, too high $\mathrm{Ca}^{2+}$ sensitivity might cause 
contraction at diastolic calcium concentrations and thereby contributes to cardiac dysfunction.

\section{Conclusion}

We show that different forms of heart failure (PPCM, DCM, and ISHD) share aspects of underlying pathomechanisms, but they also differ in important aspects. An overview of the changes in each form of heart failure is shown in Figure 6. PPCM, DCM, and ISHD have decreased cMyBP-C phosphorylation and increased $\mathrm{Ca}^{2+}$ sensitivity induced by decreased phosphorylation of cTnI. However, PPCM and DCM share additional pathomechanisms, such as increased compliant titin, reduced phosphorylation of titin at Ser4010, and impaired myofilament length-dependent activation, observations that were not seen in ISHD. The increased myofilament $\mathrm{Ca}^{2+}$ sensitivity and decreased lengthdependent activation were more pronounced in PPCM samples. Both parameters could be restored after incubation with exogenous PKA. In addition, DCM, but not PPCM, patients show induction of the expression of EH-myomesin. The lack of EH-myomesin reexpression might have contributed to an increase in $\mathrm{F}_{\text {pass }}$ and severely blunted length-dependent activation in PPCM patients in situations of low PKA-mediated phosphorylation because of instability of the sarcomeres in overstretched conditions. Therefore, this study shows myofilament remodeling and function is more impaired in PPCM, compared with DCM and ISHD, and the lack of EH-myomesin reexpression may explain the fast deterioration of some PPCM patients to endstage heart failure.

\section{Limitation to the Study}

No tissue from healthy postpartum women could be explored and, therefore, the condition of a healthy maternal heart in the postpartum phase is not known. In addition, the different heart failure groups could not be completely matched regarding age and sex of patient samples studied. However, no difference was observed between samples derived from males and females in the DCM group.

\section{Acknowledgments}

We thank Ruud Zaremba, Max Goebel, Wies Lommen, and Sabine Bongardt for technical assistance, the Sydney Heart Bank for the samples used in this study, and Dr. Mark Holt (King's College London) for help with densitometry.

\section{Supplemental Data}

Supplemental material for this article can be found at https://doi.org/10.1016/j.ajpath.2017.08.022.

\section{References}

1. Elliott P, Andersson B, Arbustini E, Bilinska Z, Cecchi F, Charron P, Dubourg O, Kuhl U, Maisch B, McKenna WJ, Monserrat L, Pankuweit S, Rapezzi C, Seferovic P, Tavazzi L, Keren A: Classification of the cardiomyopathies: a position statement from the European Society of Cardiology Working Group on Myocardial and Pericardial Diseases. Eur Heart J 2008, 29:270-276

2. Pearson GD, Veille J-C, Rahimtoola S, Hsia J, Oakley CM, Hosenpud JD, Ansari A, Baughman KL: Peripartum Cardiomyopathy National Heart, Lung, and Blood Institute and Office of Rare Diseases (National Institutes of Health) Workshop Recommendations and Review. JAMA 2000, 283:1183-1188

3. Ware JS, Li J, Mazaika E, Yasso CM, DeSouza T, Cappola TP, Tsai EJ, Hilfiker-Kleiner D, Kamiya CA, Mazzarotto F, Cook SA, Halder I, Prasad SK, Pisarcik J, Hanley-Yanez K, Alharethi R, Damp J, Hsich E, Elkayam U, Sheppard R, Kealey A, Alexis J, Ramani G, Safirstein J, Boehmer J, Pauly DF, Wittstein IS, Thohan V, Zucker MJ, Liu P, Gorcsan J 3rd, McNamara DM, Seidman CE, Seidman JG, Arany Z; IMAC-2 and IPAC Investigators: Shared genetic predisposition in peripartum and dilated cardiomyopathies. N Engl J Med 2016, 374:233-241

4. van Spaendonck-Zwarts KY, Posafalvi A, van den Berg MP, HilfikerKleiner D, Bollen IA, Sliwa K, Alders M, Almomani R, van Langen IM, van der Meer P, Sinke RJ, van der Velden J, Van Veldhuisen DJ, van Tintelen JP, Jongbloed JD: Titin gene mutations are common in families with both peripartum cardiomyopathy and dilated cardiomyopathy. Eur Heart J 2014, 35:2165-2173

5. van Spaendonck-Zwarts KY, van Tintelen JP, van Veldhuisen DJ, van der Werf R, Jongbloed JD, Paulus WJ, Dooijes D, van den Berg MP: Peripartum cardiomyopathy as a part of familial dilated cardiomyopathy. Circulation 2010, 121:2169-2175

6. Hershberger RE, Hedges DJ, Morales A: Dilated cardiomyopathy: the complexity of a diverse genetic architecture. Nat Rev Cardiol 2013, 10: $531-547$

7. Hilfiker-Kleiner D, Kaminski K, Podewski E, Bonda T, Schaefer A, Sliwa K, Forster O, Quint A, Landmesser U, Doerries C, Luchtefeld M, Poli V, Schneider MD, Balligand JL, Desjardins F, Ansari A, Struman I, Nguyen NQ, Zschemisch NH, Klein G, Heusch G, Schulz R, Hilfiker A, Drexler H: A cathepsin D-cleaved 16 $\mathrm{kDa}$ form of prolactin mediates postpartum cardiomyopathy. Cell 2007, 128:589-600

8. Sliwa K, Blauwet L, Tibazarwa K, Libhaber E, Smedema JP Becker A, McMurray J, Yamac H, Labidi S, Struman I, HilfikerKleiner D: Evaluation of bromocriptine in the treatment of acute severe peripartum cardiomyopathy: a proof-of-concept pilot study. Circulation 2010, 121:1465-1473

9. Fett JD: Peripartum cardiomyopathy: a puzzle closer to solution. World J Cardiol 2014, 6:87-99

10. Elkayam U: Clinical characteristics of peripartum cardiomyopathy in the United States: diagnosis, prognosis, and management. J Am Coll Cardiol 2011, 58:659-670

11. Felker GM, Thompson RE, Hare JM, Hruban RH, Clemetson DE, Howard DL, Baughman KL, Kasper EK: Underlying causes and long-term survival in patients with initially unexplained cardiomyopathy. N Engl J Med 2000, 342:1077-1084

12. Ballo P, Betti I, Mangialavori G, Chiodi L, Rapisardi G, Zuppiroli A: Peripartum cardiomyopathy presenting with predominant left ventricular diastolic dysfunction: efficacy of bromocriptine. Case Rep Med 2012, 2012:476903

13. Haghikia A, Podewski E, Libhaber E, Labidi S, Fischer D, Roentgen $\mathrm{P}$, Tsikas $\mathrm{D}$, Jordan $\mathrm{J}$, Lichtinghagen $\mathrm{R}$, von Kaisenberg CS, Struman I, Bovy N, Sliwa K, Bauersachs J, HilfikerKleiner D: Phenotyping and outcome on contemporary management in a German cohort of patients with peripartum cardiomyopathy. Basic Res Cardiol 2013, 108:366 
14. van Hoeven KH, Kitsis RN, Katz SD, Factor SM: Peripartum versus idiopathic dilated cardiomyopathy in young women: a comparison of clinical, pathologic and prognostic features. Int J Cardiol 1993, 40: $57-65$

15. Goland S, Modi K, Bitar F, Janmohamed M, Mirocha JM, Czer LS, Illum S, Hatamizadeh P, Elkayam U: Clinical profile and predictors of complications in peripartum cardiomyopathy. J Card Fail 2009, 15: $645-650$

16. Grewal J, Siu SC, Ross HJ, Mason J, Balint OH, Sermer M, Colman JM, Silversides CK: Pregnancy outcomes in women with dilated cardiomyopathy. J Am Coll Cardiol 2009, 55:45-52

17. Hilfiker-Kleiner D, Sliwa K: Pathophysiology and epidemiology of peripartum cardiomyopathy. Nat Rev Cardiol 2014, 11:364-370

18. Patten IS, Rana S, Shahul S, Rowe GC, Jang C, Liu L, Hacker MR, Rhee JS, Mitchell J, Mahmood F, Hess P, Farrell C, Koulisis N, Khankin EV, Burke SD, Tudorache I, Bauersachs J, del Monte F, Hilfiker-Kleiner D, Karumanchi SA, Arany Z: Cardiac angiogenic imbalance leads to peripartum cardiomyopathy. Nature 2012, 485: $333-338$

19. Grattan DR, Steyn FJ, Kokay IC, Anderson GM, Bunn SJ: Pregnancy-induced adaptation in the neuroendocrine control of prolactin secretion. J Neuroendocrinol 2008, 20:497-507

20. Stapel B, Kohlhaas M, Ricke-Hoch M, Haghikia A, Erschow S, Knuuti J, Silvola JM, Roivainen A, Saraste A, Nickel AG, Saar JA, Sieve I, Pietzsch S, Muller M, Bogeski I, Kappl R, Jauhiainen M, Thackeray JT, Scherr M, Bengel FM, Hagl C, Tudorache I, Bauersachs J, Maack C, Hilfiker-Kleiner D: Low STAT3 expression sensitizes to toxic effects of beta-adrenergic receptor stimulation in peripartum cardiomyopathy. Eur Heart J 2016, 38:349-361

21. van Dijk SJ, Paalberends ER, Najafi A, Michels M, Sadayappan S, Carrier L, Boontje NM, Kuster DW, van Slegtenhorst M, Dooijes D, dos Remedios C, ten Cate FJ, Stienen GJ, van der Velden J: Contractile dysfunction irrespective of the mutant protein in human hypertrophic cardiomyopathy with normal systolic function. Circ Heart Fail 2012, 5:36-46

22. van der Velden J, de Jong J, Owen VJ, Burton PB, Stienen GJ: Effect of protein kinase $\mathrm{A}$ on calcium sensitivity of force and its sarcomere length dependence in human cardiomyocytes. Cardiovasc Res 2000, 46:487-495

23. Warren CM, Krzesinski PR, Greaser ML: Vertical agarose gel electrophoresis and electroblotting of high-molecular-weight proteins. Electrophoresis 2003, 24:1695-1702

24. Beqqali A, Bollen IA, Rasmussen TB, van den Hoogenhof MM, van Deutekom HW, Schafer S, Haas J, Meder B, Sorensen KE, van Oort RJ, Mogensen J, Hubner N, Creemers EE, van der Velden J, Pinto YM: A mutation in the glutamate-rich region of RNA-binding motif protein 20 causes dilated cardiomyopathy through missplicing of titin and impaired Frank-Starling mechanism. Cardiovasc Res 2016, 112:452-463

25. Ottenheijm CA, Knottnerus AM, Buck D, Luo X, Greer K, Hoying A, Labeit S, Granzier H: Tuning passive mechanics through differential splicing of titin during skeletal muscle development. Biophys J 2009, 97:2277-2286

26. Kötter S, Kazmierowska M, Andresen C, Bottermann K, Grandoch M, Gorressen S, Heinen A, Moll JM, Scheller J, Gödecke A, Fischer JW, Schmitt JP, Krüger M: Titin-based cardiac myocyte stiffening contributes to early adaptive ventricular remodeling after myocardial infarction. Circ Res 2016, 119:1017-1029

27. Zaremba R, Merkus D, Hamdani N, Lamers JM, Paulus WJ, Dos Remedios C, Duncker DJ, Stienen GJ, van der Velden J: Quantitative analysis of myofilament protein phosphorylation in small cardiac biopsies. Proteomics Clin Appl 2007, 1:1285-1290

28. Najafi A, Schlossarek S, van Deel ED, van den Heuvel N, Guclu A, Goebel M, Kuster DW, Carrier L, van der Velden J: Sexual dimorphic response to exercise in hypertrophic cardiomyopathy-associated MYBPC3-targeted knock-in mice. Pflugers Arch 2015, 467:1303-1317

29. Avner BS, Shioura KM, Scruggs SB, Grachoff M, Geenen DL, Helseth DL Jr, Farjah M, Goldspink PH, Solaro RJ: Myocardial infarction in mice alters sarcomeric function via post-translational protein modification. Mol Cell Biochem 2012, 363:203-215

30. Kaestner L, Scholz A, Hammer K, Vecerdea A, Ruppenthal S, Lipp P: Isolation and genetic manipulation of adult cardiac myocytes for confocal imaging. J Vis Exp 2009, 31:e1433

31. Grove BK, Kurer V, Lehner C, Doetschman TC, Perriard JC, Eppenberger HM: A new 185,000-dalton skeletal muscle protein detected by monoclonal antibodies. J Cell Biol 1984, 98:518-524

32. Schoenauer R, Emmert MY, Felley A, Ehler E, Brokopp C, Weber B, Nemir M, Faggian GG, Pedrazzini T, Falk V, Hoerstrup SP, Agarkova I: EH-myomesin splice isoform is a novel marker for dilated cardiomyopathy. Basic Res Cardiol 2011, 106:233-247

33. Granzier $\mathrm{H}$, Labeit $\mathrm{S}$ : Cardiac titin: an adjustable multi-functional spring. J Physiol 2002, 541:335-342

34. Fukuda N, Terui T, Ohtsuki I, Ishiwata S, Kurihara S: Titin and troponin: central players in the frank-starling mechanism of the heart. Curr Cardiol Rev 2009, 5:119-124

35. Fukuda N, Wu Y, Farman G, Irving TC, Granzier H: Titin isoform variance and length dependence of activation in skinned bovine cardiac muscle. J Physiol 2003, 553:147-154

36. Makarenko I, Opitz CA, Leake MC, Neagoe C, Kulke M, Gwathmey JK, del Monte F, Hajjar RJ, Linke WA: Passive stiffness changes caused by upregulation of compliant titin isoforms in human dilated cardiomyopathy hearts. Circ Res 2004, 95:708-716

37. Nagueh SF, Shah G, Wu Y, Torre-Amione G, King NM, Lahmers S, Witt CC, Becker K, Labeit S, Granzier HL: Altered titin expression, myocardial stiffness, and left ventricular function in patients with dilated cardiomyopathy. Circulation 2004, 110:155-162

38. Seddon M, Looi YH, Shah AM: Oxidative stress and redox signalling in cardiac hypertrophy and heart failure. Heart 2007, 93: 903-907

39. Alegre-Cebollada J, Kosuri P, Giganti D, Eckels E, Rivas-Pardo JA, Hamdani N, Warren CM, Solaro RJ, Linke WA, Fernandez JM: Sglutathionylation of cryptic cysteines enhances titin elasticity by blocking protein folding. Cell 2014, 156:1235-1246

40. Stathopoulou K, Wittig I, Heidler J, Piasecki A, Richter F, Diering S, van der Velden J, Buck F, Donzelli S, Schroder E, Wijnker PJ, Voigt N, Dobrev D, Sadayappan S, Eschenhagen T, Carrier L, Eaton P, Cuello F: S-glutathiolation impairs phosphoregulation and function of cardiac myosin-binding protein $\mathrm{C}$ in human heart failure. FASEB J 2016, 30:1849-1864

41. Harding S, Brown L, Wynne D, Davies C, Poole-Wilson P: Mechanisms of beta adrenoceptor desensitisation in the failing human heart. Cardiovasc Res 1994, 28:1451-1460

42. Kötter S, Gout L, Von Frieling-Salewsky M, Muller AE, Helling S, Marcus K, Dos Remedios C, Linke WA, Kruger M: Differential changes in titin domain phosphorylation increase myofilament stiffness in failing human hearts. Cardiovasc Res 2013, 99: 648-656

43. Wijnker PJM, Sequeira V, Foster DB, Li Y, dos Remedios CG, Murphy AM, Stienen GJM, van der Velden J: Length-dependent activation is modulated by cardiac troponin I bisphosphorylation at Ser23 and Ser24 but not by Thr143 phosphorylation. Am J Physiol Heart Circ Physiol 2014, 306:H1171-H1181

44. Konhilas JP, Irving TC, Wolska BM, Jweied EE, Martin AF, Solaro RJ, de Tombe PP: Troponin I in the murine myocardium: influence on length-dependent activation and interfilament spacing. J Physiol 2003, 547:951-961

45. Assomull RG, Prasad SK, Lyne J, Smith G, Burman ED, Khan M, Sheppard MN, Poole-Wilson PA, Pennell DJ: Cardiovascular magnetic resonance, fibrosis, and prognosis in dilated cardiomyopathy. J Am Coll Cardiol 2006, 48:1977-1985

46. Herpel E, Pritsch M, Koch A, Dengler TJ, Schirmacher P, Schnabel PA: Interstitial fibrosis in the heart: differences in extracellular matrix proteins and matrix metalloproteinases in end-stage dilated, ischaemic and valvular cardiomyopathy. Histopathology 2006, 48:736-747 
47. Kawano H, Tsuneto A, Koide Y, Tasaki H, Sueyoshi E, Sakamoto I, Hayashi T: Magnetic resonance imaging in a patient with peripartum cardiomyopathy. Intern Med 2008, 47:97-102

48. Leurent G, Baruteau AE, Larralde A, Ollivier R, Schleich JM, Boulmier D, Bedossa M, Langella B, Le Breton H: Contribution of cardiac MRI in the comprehension of peripartum cardiomyopathy pathogenesis. Int J Cardiol 2009, 132:e91-e93

49. Mouquet F, Lions C, de Groote P, Bouabdallaoui N, Willoteaux S, Dagorn J, Deruelle P, Lamblin N, Bauters C, Beregi JP: Characterisation of peripartum cardiomyopathy by cardiac magnetic resonance imaging. Eur Radiol 2008, 18:2765-2769

50. Ntusi NBA, Chin A: Letter to the editor: characterisation of peripartum cardiomyopathy by cardiac magnetic resonance imaging. Eur Radiol 2009, 19:1324-1325

51. Ricke-Hoch M, Bultmann I, Stapel B, Condorelli G, Rinas U, Sliwa K, Scherr M, Hilfiker-Kleiner D: Opposing roles of Akt and STAT3 in the protection of the maternal heart from peripartum stress. Cardiovasc Res 2014, 101:587-596

52. Hilfiker-Kleiner D, Haghikia A, Nonhoff J, Bauersachs J: Peripartum cardiomyopathy: current management and future perspectives. Eur Heart J 2015, 36:1090-1097

53. Methawasin M, Hutchinson KR, Lee EJ, Smith JE 3rd, Saripalli C, Hidalgo CG, Ottenheijm CA, Granzier H: Experimentally increasing titin compliance in a novel mouse model attenuates the Frank-Starling mechanism but has a beneficial effect on diastole. Circulation 2014, 129:1924-1936

54. Agarkova I, Auerbach D, Ehler E, Perriard J-C: A novel marker for vertebrate embryonic heart, the EH-myomesin isoform. J Biol Chem 2000, 275:10256-10264
55. Lahmers S, Wu Y, Call DR, Labeit S, Granzier H: Developmental control of titin isoform expression and passive stiffness in fetal and neonatal myocardium. Circ Res 2004, 94:505-513

56. Freiburg A, Trombitas K, Hell W, Cazorla O, Fougerousse F, Centner T, Kolmerer B, Witt C, Beckmann JS, Gregorio CC, Granzier H, Labeit S: Series of exon-skipping events in the elastic spring region of titin as the structural basis for myofibrillar elastic diversity. Circ Res 2000, 86:1114-1121

57. Agarkova I, Schoenauer R, Ehler E, Carlsson L, Carlsson E, Thornell L-E, Perriard J-C: The molecular composition of the sarcomeric M-band correlates with muscle fiber type. Eur J Cell Biol 2004, 83:193-204

58. Thum T, Galuppo P, Wolf C, Fiedler J, Kneitz S, van Laake LW, Doevendans PA, Mummery CL, Borlak J, Haverich A, Gross C, Engelhardt S, Ertl G, Bauersachs J: MicroRNAs in the human heart: a clue to fetal gene reprogramming in heart failure. Circulation 2007, $116: 258-267$

59. Schoenauer R, Bertoncini P, Machaidze G, Aebi U, Perriard JC, Hegner M, Agarkova I: Myomesin is a molecular spring with adaptable elasticity. J Mol Biol 2005, 349:367-379

60. Obermann WMJ, Gautel M, Weber K, Fürst DO: Molecular structure of the sarcomeric $\mathrm{M}$ band: mapping of titin and myosin binding domains in myomesin and the identification of a potential regulatory phosphorylation site in myomesin. EMBO J 1997, 16: $211-220$

61. Fukuzawa A, Lange S, Holt M, Vihola A, Carmignac V, Ferreiro A, Udd B, Gautel M: Interactions with titin and myomesin target obscurin and obscurin-like 1 to the M-band: implications for hereditary myopathies. J Cell Sci 2008, 121:1841-1851 